Article

\title{
Synthesis of Dihydropyrano[3,2-c]pyrazoles via Double Bond Migration and Ring-Closing Metathesis
}

\author{
Yoshihide Usami *(D), Kodai Sumimoto, Azusa Kishima, Yuya Tatsui, Hiroki Yoneyama and \\ Shinya Harusawa \\ Department of Pharmaceutical Organic Chemistry, Osaka University of Pharmaceutical Sciences, 4-20-1 \\ Nasahara, Takatsuki, Osaka 569-1094, Japan; e12241@gap.oups.ac.jp (K.S.); e13307@gap.oups.ac.jp (A.K.); \\ e18902@gap.oups.ac.jp (Y.T.); yoneyama@gly.oups.ac.jp (H.Y.); harusawa@gly.oups.ac.jp (S.H.) \\ * Correspondence: usami@gly.oups.ac.jp; Tel.: +81-726-90-1087
}

Received: 25 December 2018; Accepted: 11 January 2019; Published: 15 January 2019

\begin{abstract}
Three types of pyrazole-fused heterobicycles, i.e., 1,5-, 1,7-, and 2,5-dihydropyrano[3,2-c] pyrazoles, were synthesized from 4-allyloxy- $1 H$-pyrazoles. A sequence of the Claisen rearrangement of 4-allyloxy- $1 \mathrm{H}$-pyrazoles, ruthenium-hydride-catalyzed double bond migration, $\mathrm{O}$-allylation, and ring-closing metathesis was employed in this study.
\end{abstract}

Keywords: dihydropyrano[3,2-c]pyrazole; synthesis; double bond migration; ruthenium hydride catalyst; ring-closing metathesis

\section{Introduction}

The synthesis of substituted or functionalized pyrazoles has been studied extensively thus far because they show or are expected to show important and diverse bioactivities [1,2]. Celecoxib, a non-steroidal anti-inflammatory drug (NSAID), is a representative pyrazole-containing compound, which acts through selective cyclooxygenase (COX)-2 inhibition. Whereas the late-stage construction of a pyrazole ring through some cycloadditions of already-substituted components is the basis for most syntheses of substituted pyrazoles [3,4], direct functionalization of pyrazoles has not been investigated satisfactorily to date. As investigations on it seem rare, we have been interested in and studied the direct functionalization of pyrazoles through coupling reactions of halogenated analogues derived from commercially available pyrazole [5-8]. In addition, pyrazole-fused heterocycles have recently been synthesized for reasons similar to those described above or because of characteristic activities not seen in monocyclic substituted pyrazoles [9]. Many pyrazole-fused heterocyclic compounds possess unique and important biological activities [10]. Some examples of pyrano[2,3-c]pyrazoles [11-14], pyrano[3,2-c]pyrazoles $[15,16]$, and furo[3,2-c]pyrazoles [17,18] are presented in Figure 1.

The Claisen rearrangement followed by ring-closing metathesis (RCM) is an effective sequence for constructing various polycyclic systems $[19,20]$. On the basis of our previous work on the synthesis of withasomnines [21,22], we recently reported the synthesis of dihydrooxepino[3,2-c]pyrazoles (4 and its isomers) via a combination of the Claisen rearrangement of 4-allyloxy- $1 H$-pyrazoles (1a-d), $\mathrm{O}$-allylation of Claisen rearrangement product $\mathbf{2}$ into 3, and subsequent RCM of 3 [23]. This realized the construction of pyrazole-containing 5,7-bicyclic system 4, shown in Scheme 1. 
<smiles>CNC(=O)c1nn(-c2ccccc2)c2oc(=O)cc(C)c12</smiles>

analgesic agent ${ }^{11}$<smiles>CC(=O)c1nn(Cc2ccccc2)c2oc(=O)cc(C)c12</smiles>

antiplatelet agent ${ }^{12}$<smiles>Cc1[nH]nc2c1C(c1ccccc1)C(C#N)=C(N)O2</smiles>

anticancer agent $^{13}$<smiles>COc1cc(OCc2ccccc2)cc(C2C(C#N)=C(N)Oc3n[nH]c(C)c32)c1</smiles>

$\alpha$-glucosidase inhibitor ${ }^{14}$<smiles>CC(C)(C)n1cc2c(n1)C(=O)CC1(CCN(C(=O)c3ccc4cn[nH]c4c3)CC1)O2</smiles>

acetyl CoA carboxylase inhibitor ${ }^{15}$<smiles>O=c1c2ccccc2oc2cnn(-c3ccccc3)c12</smiles>

benzodiazepine receptor binding agent ${ }^{16}$<smiles>Cc1cc2c(o1)c(-c1ccc(CO)o1)nn2Cc1ccccc1</smiles>

anti-leukemia agent ${ }^{17}$<smiles>Cc1cc2c(o1)c(-c1ccc(C(=O)O)o1)nn2Cc1ccccc1</smiles>

neuroprotectant ${ }^{18}$<smiles>Cc1cc2c(o1)c(-c1ccc(CO)cc1)nn2-c1ccccc1</smiles>

neuroprotectant ${ }^{18}$

Figure 1. Examples of bioactive pyrano[2,3-c]pyrazoles, pyrano[3,2-c]pyrazoles, and furo[3,2-c]pyrazoles.

After the migration of the double bond in the side chain of intermediate 2 in Scheme 1, expected product 5 can be $O$-allylated to 6 . The subsequent RCM of 6 may provide a pyrazole-containing 5,6-bicyclic system, i.e., a dihydropyrano[3,2-c]pyrazole. These are expected to show various types of activities. There have been many reports of syntheses of pyrano[2,3-c]pyrazoles [10-14], but very few for pyrano[3,2-c]pyrazoles $[15,16,24,25]$. In addition, the development of a new synthetic method for furo[3,2-c]pyrazoles, which are extremely important as mentioned above, seems possible if both double bond migration and dehydrohalogenation occur on a 5-allyl-4-(2-haloethyl)oxy-1H-1-tritylpyrazole. Described herein is a new and selective synthesis of three types of dihydropyrano[3,2-c]pyrazoles, namely 7, 8, and 20, with pyrazole-fused heterocyclic skeletons from 1 via the combination of Claisen rearrangements and RCM, along with efforts toward furo[3,2-c]pyrazoles (17). 


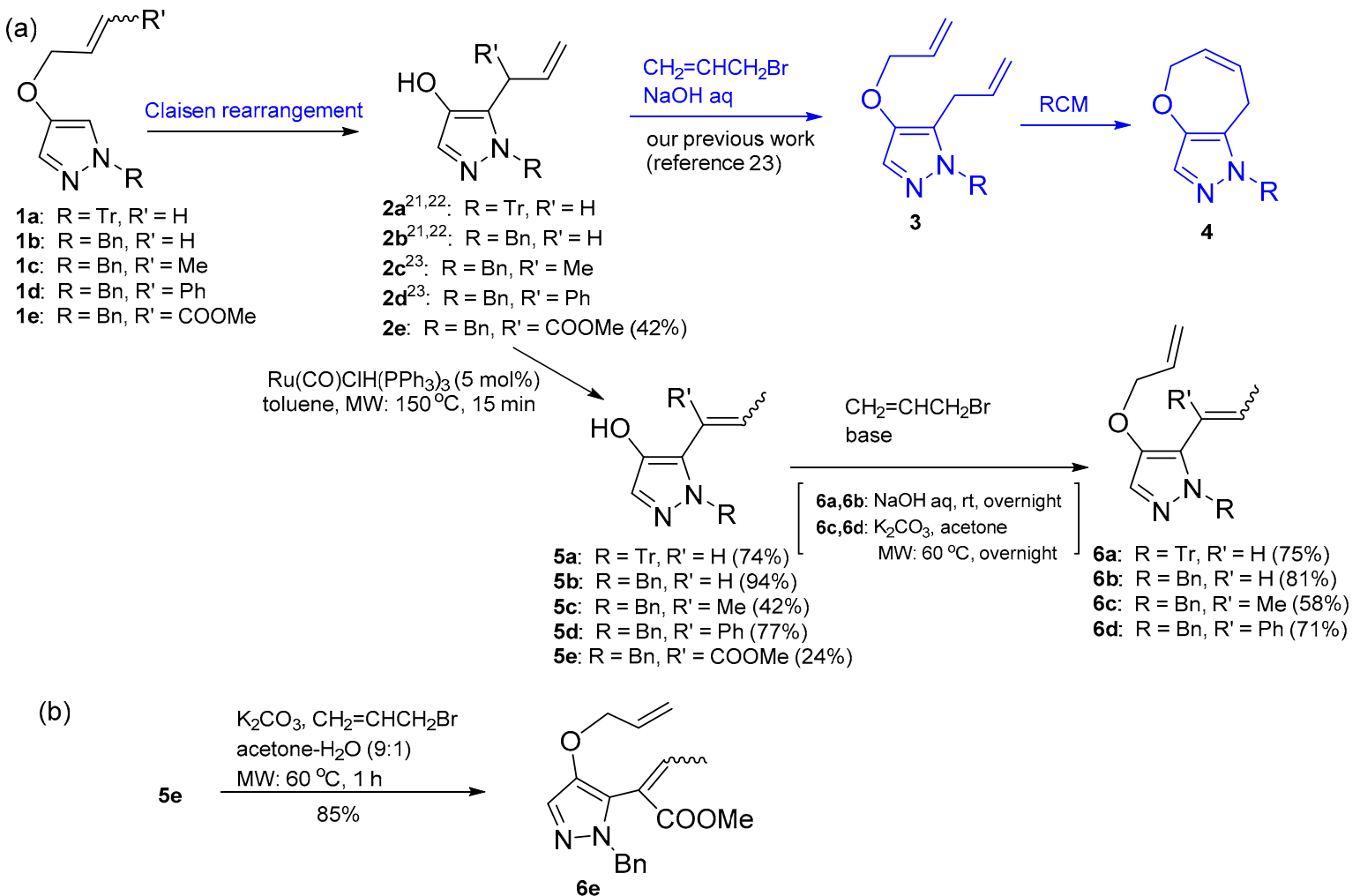

Scheme 1. Preparation of 5-allyl-4-allyloxy- $1 H$-pyrazoles (6) from 4-allyloxy-1H-pyrazoles (2).

\section{Results}

\subsection{Synthesis of 1,5-Dihydropyrano[3,2-c]pyrazoles}

Our initial efforts in the synthesis of 1,5-dihydropyrano[3,2-c]pyrazoles (7) are presented in Scheme 1 and Table 1. In our earlier efforts for double bond migration for the conversion of $\mathbf{2 a}$ to $\mathbf{5 a}$ with potassium tert-butoxide $(t-\mathrm{BuOK})$ as a base, every trial under microwave (MW) irradiation in a different solvent (tetrahydrofuran (THF), EtOH, $\mathrm{MeCN}$, acetone, 1,2-dimethoxyethane (DME), toluene, THF-toluene) failed to give the desired product 5a [20,26]. Alternatively, carbonylchlorohydridotris(triphenylphosphine)ruthenium(II) $\left[\left(\mathrm{RuClH}(\mathrm{CO})(\mathrm{PPh})_{3}\right]\right.$ was applied to the double bond migration for the conversion of $\mathbf{2}$ to $\mathbf{5}$, as shown in Scheme 1 [27]. MW irradiation of the reaction mixture of 2 and $5 \mathrm{~mol} \%$ of the ruthenium hydride catalyst in toluene gave the desired product 5, whereas the same reaction at room temperature (rt) did not occur. Starting compounds $\mathbf{2 a - d}$ are known compounds [21-23], and 1-benzyl-4-hydroxy-5-((1-methoxycarbonyl)-2-propen-1-yl)-1H-pyrazole (2e) is the Claisen rearrangement product of $\mathbf{1 e}$, which was newly prepared from 1-benzyl-4-iodo-1H-pyrazole for this work and already contained a small part of $5 \mathbf{e}$ (see Experimental section).

Then, the C4-hydroxyl groups in 4-hydroxy-5-(1-propenyl)-1H-pyrazoles $\mathbf{5 a}$ and $\mathbf{5} \mathbf{b}$ were treated with aqueous $\mathrm{NaOH}$ followed by alkenyl halides in order to prepare the RCM substrates $\mathbf{6 a}$ and $\mathbf{6 b}$. Conversion of $\mathbf{5 c}$ and $\mathbf{5 d}$, which have a substituent, to $\mathbf{6 c}$ and $\mathbf{6 d}$ using the same condition took a long time with poor yields. So, alternative transformation of $\mathbf{5 c}$ and $\mathbf{5 d}$ to $\mathbf{6 c}$ and $\mathbf{6 d}$ was carried out using $\mathrm{K}_{2} \mathrm{CO}_{3}$ in acetone under $\mathrm{MW}$ irradiation, respectively. The reactions proceeded smoothly and the chemical yields of $\mathbf{6} \mathbf{c}$ and $\mathbf{6} \mathbf{d}$ are presented in Scheme 1a. In a separate experiment, compound $\mathbf{2 e}$, which already contains a small part of $\mathbf{5 e}$ as noted above, was transformed directly to $6 \mathbf{e}$ through treatment with $\mathrm{K}_{2} \mathrm{CO}_{3}$ and allyl bromide in acetone under MW irradiation in $63 \%$ yield, since the yield from $2 \mathbf{e}$ to $5 \mathbf{e}$ was not satisfactory. The yield of the MW-aided transformation of $\mathbf{2} \mathbf{e}$ to $6 \mathbf{e}$ was improved to $85 \%$ by applying acetone-water (9:1) as the solvent system (Scheme $1 \mathrm{~b}$ ). 
Table 1. Ring-closing metathesis (RCM) of 5-allyl-4-allyloxy- $1 H$-pyrazoles.

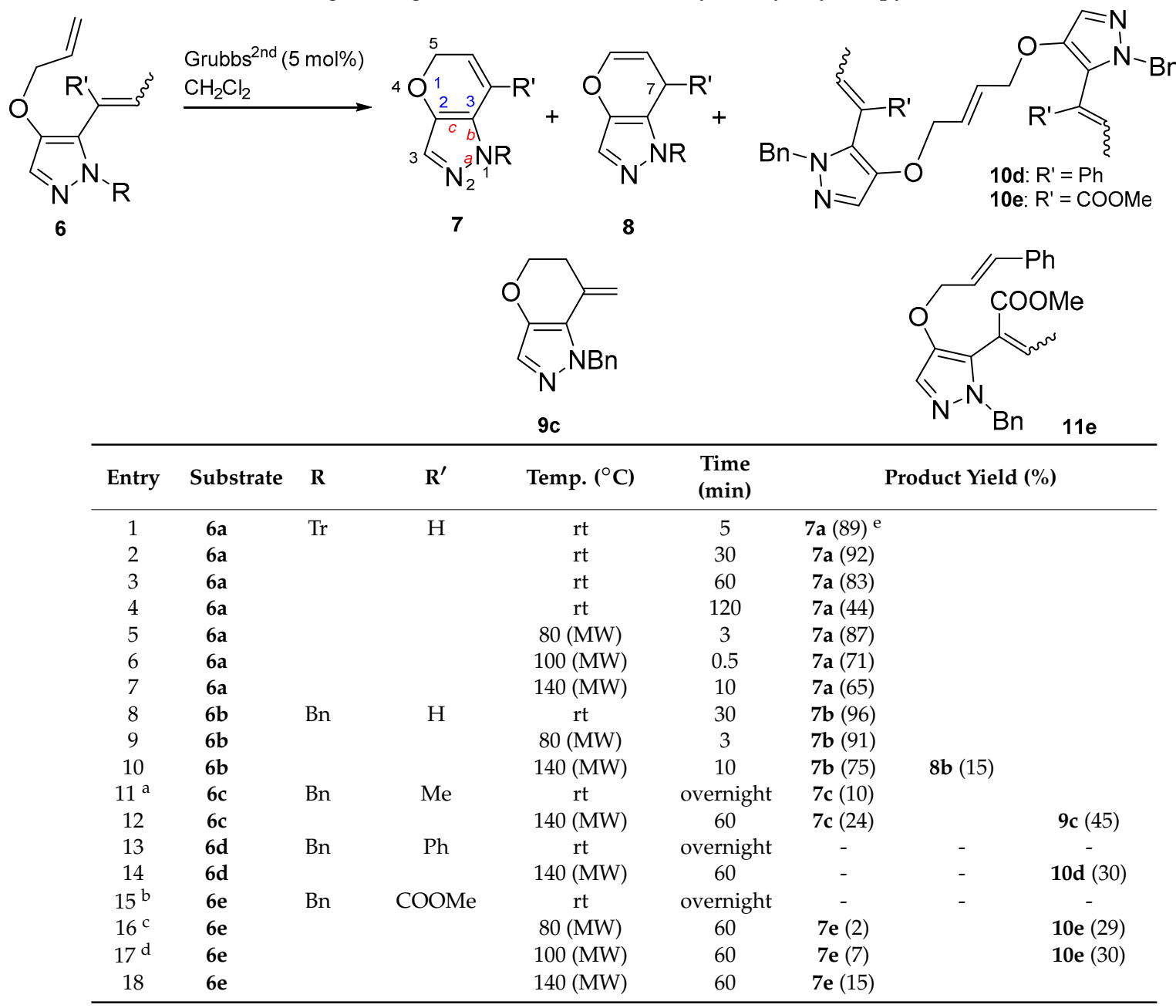

a. $60 \%$ of starting material 6 c was recovered. b. $50 \%$ of 6 e was recovered. c. Undesired 11 e $(2 \%)$ was obtained during the recovery of $6 \mathbf{e}(21 \%)$. d. 11e was obtained $(4 \%)$. e. A small amount of $6 \mathbf{a}$ was detected in the NMR spectrum and was inseparable from $7 \mathbf{a}$.

RCM substrates 6 were treated with $5 \mathrm{~mol} \%$ Grubbs $^{\prime}$ second-generation catalyst (Grubbs ${ }^{2 n d}$ ) in $\mathrm{CH}_{2} \mathrm{Cl}_{2}$. The results of the $\mathrm{RCM}$ reactions are summarized in Table 1 . With substrate $6 \mathbf{a}$, reaction at $\mathrm{rt}$ afforded the desired RCM product 7a within $30 \mathrm{~min}$ (entry 2). A shorter reaction time also led to 7a, but with an inseparable trace amount of $\mathbf{6 a}$ (entry 1). In contrast, extended reaction times led to reduced product yields (entries 3 and 4). The MW-aided reaction was also examined in an attempt to reduce the reaction time (entries 5-7). In these trials, only 7a was formed and double bond migration product 8a could not be detected [23]. Moreover, higher temperatures above $100{ }^{\circ} \mathrm{C}$ reduced the reaction yield (entry 7). The optimal reaction conditions in entries 2 and 5 for substrate 6 a were applied to the RCM of $\mathbf{6 b}$ and gave similar results producing $7 \mathbf{b}$ (entries 8 and 9 , respectively). The MW reaction of $6 \mathbf{b}$ at a higher temperature of $140{ }^{\circ} \mathrm{C}$ led to partial double bond migration to produce $8 \mathbf{b}$ (entry 10). When the substrate had an $\mathrm{R}^{\prime}$ substituent, different results were obtained, as shown by the following entries. Substrates $\mathbf{6 d}$ and $\mathbf{6 e}$ did not react at $\mathrm{rt}$ (entries 13 and 15, respectively).

The MW-aided reaction $\left(140^{\circ} \mathrm{C}\right)$ of $6 \mathrm{c}$ afforded RCM product $7 \mathrm{c}$ as a minor product $(24 \%)$ and 9c $(45 \%)$ with an exomethylene moiety as the major product (entry 12). The structure of 9c was determined through the heteronuclear single quantum coherence (HSQC) correlations between a carbon signal at $\delta 107.2 \mathrm{ppm}$ and two proton signals at $\delta 4.78$ and $4.96 \mathrm{ppm}$. Generally, endo-cyclic alkene is considered to be more stable than the corresponding exo-alkene. But in this case, $7 \mathrm{c}$ is thought 
to be less stable than exo-diene $9 \mathrm{c}$ due to the strain caused by 6-membered endo-diene structure in the thermodynamic condition.

However, the same MW conditions applied to substrate $\mathbf{6 d}$ did not result in $\mathbf{7 d}$, but dimeric $\mathbf{1 0 d}$ formed through intermolecular metathesis in $30 \%$ yield (entry 14). Mass spectrometry (MS) revealed that compound $10 \mathrm{~d}$ had an $m / z$ of $632\left(\mathrm{M}^{+}\right)$, which corresponds to $\mathrm{C}_{42} \mathrm{H}_{42} \mathrm{~N}_{4} \mathrm{O}_{2}$. The ${ }^{1} \mathrm{H}$ nuclear magnetic resonance (NMR) spectrum of $10 \mathrm{~d}$ suggested the presence of a $=\mathrm{CHCH}_{3}$ moiety through the signals at $\delta 6.29(\mathrm{q}, J=7.1 \mathrm{~Hz})$ and $1.51 \mathrm{ppm}(\mathrm{d}, J=7.1 \mathrm{~Hz})$ in a 1:3 integral ratio and the lack of an exomethylene from the starting $\mathbf{6 d}$. These data suggest that the intermolecular metathesis product $\mathbf{1 0 d}$ formed by expelling an ethylene molecule $\left[339(\mathbf{6 d}) \times 2-28\left(\mathrm{CH}_{2}=\mathrm{CH}_{2}\right)=632\left(\left(\mathrm{M}^{+}\right)\right.\right.$for $\left.\left.10 \mathrm{~d}\right)\right]$. The presence of a bulky $\mathrm{R}^{\prime}$ substituent may lead to serious repulsion in the transition state for RCM. When the substrate had a methoxycarbonyl group as $\mathrm{R}^{\prime}$, the results were confusing. The MW reaction of $6 \mathrm{e}$ at $140{ }^{\circ} \mathrm{C}$ gave a complex mixture and only $7 \mathbf{e}$ was isolated in $15 \%$ yield (entry 18$)$. The MW reactions of $6 \mathbf{e}$ at lower temperatures $\left(80\right.$ and $100{ }^{\circ} \mathrm{C}$ ) gave $10 \mathbf{e}$ in similar yields $(29 \%$ and $30 \%$, respectively) with $\mathbf{7 e}$ as a minor product (entries 16 and 17). In both of these entries, 11e, which is a metathesis product of $\mathbf{6 e}$ and the Grubbs catalyst, was also isolated as a minor product. The structure of 11e was confirmed through detailed NMR analysis and an $\mathrm{M}^{+}$peak at $m / z 388.1785\left(\mathrm{C}_{24} \mathrm{H}_{24} \mathrm{~N}_{2} \mathrm{O}_{3}\right)$ in the high-resolution MS (HRMS) spectrum. However, our attention was focused on increasing the yields of $7 \mathrm{e}$ and decreasing the yields of $10 \mathrm{e}$ by increasing the reaction temperature (entries 16-18). Then, we hypothesized that $10 \mathbf{e}$ transforms into $7 \mathbf{e} ; \mathbf{1 0 e}$ may be the initial product at lower reaction temperatures. Therefore, the MW reaction of pure 10e with Grubbs ${ }^{2 \text { nd }}$ at $140{ }^{\circ} \mathrm{C}$ was examined independently in an attempt to observe the formation of $\mathbf{7 e}$ as the major product in the reaction mixture.

\subsection{Synthesis of 1,7-Dihydropyrano[3,2-c]pyrazoles}

We attempted to expand this methodology to the syntheses of different types of pyrazole-fused heterobicycles, i.e., 1,7-dihydropyrano[3,2-c]pyrazoles (8) and furo[3,2-c] pyrazoles (17), as illustrated in Scheme 2. In order to realize this, 4-O-vinylation was required. First, the 4-hydroxyl group of 2a was treated with 1,2-dichloroethane to obtain a pyrazole with a 2-chloroethoxy group at $\mathrm{C} 4, \mathbf{1 2}_{\mathrm{Cl}}$. However, dehydrochlorination of $\mathbf{1 2}_{\mathrm{Cl}}$ did not occur under basic conditions. Then, 2-bromoethylation of the 4-hydroxyl group was examined, aimed at improving the leaving ability. Desired 5-allyl-4-(2-bromoethyl)oxy-1H-1-tritylpyrazole (12a) was smoothly prepared through the MW-aided reaction of 2a. The examination of the dehydrobromination of 12a is summarized in Table 2. Whereas treatment of 12a with $t$-BuOK in toluene resulted in no reaction (entry 1), application of THF-MeOH (4:1) led to the desired dehydrobromination (entries 2-5). The MW reaction at $100{ }^{\circ} \mathrm{C}$ for 30 min afforded only double bond migration product (E/Z)-5-allyl-4-vinyloxy-1H-1-tritylpyrazole (13a) but in 14\% yield (entry 2). Increasing the reaction time to $60 \mathrm{~min}$ resulted in an inseparable mixture of 13a and 5-(1-propenyl)-4-vinyloxy-1H-1-tritylpyrazole (14a) in 19\% combined yield (entry 3). A higher temperature of $130{ }^{\circ} \mathrm{C}$ resulted in only 14a in $30 \%$ yield (entry 4). A similar MW reaction at $80^{\circ} \mathrm{C}$ produced 13a in a similar yield (entry 5). In these trials (entries 2-5), the chemical yields of desired 13a and 14a were not satisfactory. Close inspection of entries 4 and 5 led us to isolate and elucidate the structures of side product 15 ( $28 \%$ yield), which should have formed via $\mathrm{S}_{\mathrm{N}} 2$ attack by a methoxide on 12a, and $\mathbf{1 6}$ (17\% yield) (see footnotes of Table 2). To improve the chemical yields, inhibition of the $\mathrm{S}_{\mathrm{N}} 2$ attack on 12a by a nucleophile formed from the solvent under basic conditions was required. Hence, $t-\mathrm{BuOH}$ was applied instead of $\mathrm{MeOH}$ as a co-solvent. Although the MW reaction at $80^{\circ} \mathrm{C}$ afforded only a trace amount of desired product $13 \mathrm{a}$ (entry 6 ), the same reaction at $130{ }^{\circ} \mathrm{C}$ afforded only $13 \mathrm{a}$ in $87 \%$ yield (entry 7). Inspired by the result in entry 4 , the MW reaction was attempted at a higher temperature of $180{ }^{\circ} \mathrm{C}$ and afforded 14a selectively in $67 \%$ yield (entry 8 ). Treatment of the $N$-benzyl derivative $\mathbf{1 2 b}$ with $t$-BuOK at $130{ }^{\circ} \mathrm{C}$ resulted in only $\mathbf{1 4 b}$ (72\%) (entry 9 ). Then, the dehydrobromination of $\mathbf{1 2} \mathbf{b}$ was examined at a lower temperature (entry 10), but resulted in an inseparable mixture of $\mathbf{1 2 b}$ and $\mathbf{1 4 b}$. 


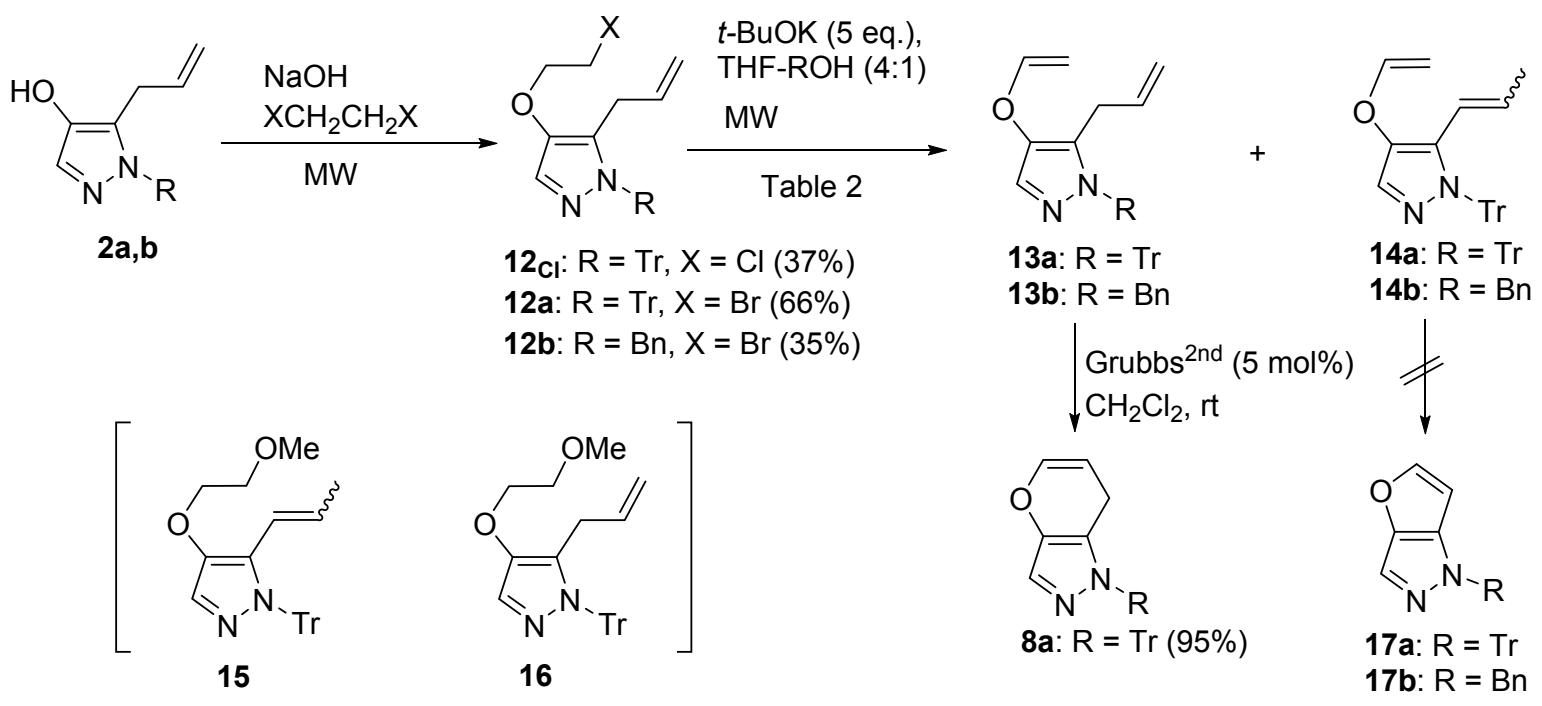

Scheme 2. Challenges in the syntheses of 1,7-dihydropyrano[3,2-c]pyrazoles (8) and furo[3,2-c]pyrazoles (19).

Table 2. Potassium $t$-butoxide promoted dehydrohalogenation of $\mathbf{1 2}$.

\begin{tabular}{ccccccc}
\hline Entry & Substrate & Solvent & Time (min) & Temp. $\left({ }^{\circ} \mathbf{C}\right)$ & \multicolumn{2}{c}{ Product Yield (\%) } \\
\hline 1 & $\mathbf{1 2 a}$ & THF & 30 & 100 & \multicolumn{2}{c}{ No reaction } \\
2 & $\mathbf{1 2 a}$ & THF:MeOH $(4: 1)$ & 30 & 100 & $\mathbf{1 3 a}(14)$ & $\mathbf{1 4 a}(0)$ \\
3 & $\mathbf{1 2 a}$ & THF:MeOH $(4: 1)$ & 60 & 100 & $\mathbf{1 3 a}+\mathbf{1 4 a}(19)^{\mathrm{a}}$ \\
$4^{\mathrm{b}}$ & $\mathbf{1 2 a}$ & THF:MeOH $(4: 1)$ & 60 & 130 & $\mathbf{1 3 a}(0)$ & $\mathbf{1 4 a}(30)$ \\
$5^{\mathrm{c}}$ & $\mathbf{1 2 a}$ & THF:MeOH $(4: 1)$ & 60 & 80 & $\mathbf{1 3 a}(27)$ & $\mathbf{1 4 a}(0)$ \\
6 & $\mathbf{1 2 a}$ & THF: $t$-BuOH $(4: 1)$ & 60 & 80 & $\mathbf{1 3 a}($ trace) & $\mathbf{1 4 a}(0)$ \\
7 & $\mathbf{1 2 a}$ & THF: $t-\mathrm{BuOH}(4: 1)$ & 60 & 130 & $\mathbf{1 3 a}(87)$ & $\mathbf{1 4 a}(0)$ \\
8 & $\mathbf{1 2 a}$ & THF: $t-\mathrm{BuOH}(4: 1)$ & 60 & 180 & $\mathbf{1 3 a}(0)$ & $\mathbf{1 4 a}(67)$ \\
9 & $\mathbf{1 2 b}$ & THF: $t-\mathrm{BuOH}(4: 1)$ & 60 & 130 & $\mathbf{1 3 b}(0)$ & $\mathbf{1 4 b}(72)$ \\
$10^{\mathrm{d}}$ & $\mathbf{1 2 b}$ & THF: $t-\mathrm{BuOH}(4: 1)$ & 60 & 80 & $\mathbf{1 3 b}(0)$ & $\mathbf{1 4 b}(31) \mathrm{e}$ \\
\hline
\end{tabular}

a. Combined yield of 13a and 14a. b. Formation of side product $15(28 \%)$ was observed. c. Formation of side product $16(17 \%)$ was observed. d. An inseparable mixture of $\mathbf{1 2 b}$ and $\mathbf{1 4 b}$ was obtained. e. Combined yields of $(E)-\mathbf{1 4 b}(25 \%)$ and $(Z)-\mathbf{1 4 b}(6 \%)$ calculated from the ${ }^{1} \mathrm{H}$ NMR spectrum with unreacted $\mathbf{1 2 b}(6 \%)$.

The RCM of prepared substrates 13a, 14a, and 14b were examined. Treatment of 13a with Grubbs $^{2 \mathrm{nd}}(5 \mathrm{~mol} \%)$ at $\mathrm{rt}$ gave the desired product $\mathbf{8 a}$ in $95 \%$ yield. However, the corresponding reactions of $14 a$ and $14 b$ did not afford the desired products $17 a$ and $17 b$, even with MW assistance. Further examinations of 14a with alternative catalysts, such as the Grubbs ${ }^{1 s t}$, Hoveyda-Grubbs, and Schrock catalysts, also did not lead to $\mathbf{1 7 a}$. Our synthesis of $\mathbf{1 7}$ will be continued in a future study.

\subsection{Synthesis of 2,5-Dihydropyrano[3,2-c]pyrazoles}

The synthesis of 2,5-dihydropyrano[3,2-c]pyrazoles (20) was examined and the results are summarized in Scheme 3. For this purpose, selective preparation of 3-alkenyl-4-allyloxy-1H-pyrazoles 19 is required since 3-allyl-4-hydroxy-1H-1-tritylpyrazole is a minor Claisen rearrangement product of 1a, and the corresponding 3-allyl-1-benzyl-4-hydroxy- $1 H$-pyrazole could not be obtained by heating $\mathbf{1 b}[21,22]$. Hence, an alternative method of preparing $\mathbf{1 9}$ via a deprotection-reprotection sequence was examined. 4-Allyloxy-5-(1-propenyl)-1H-1-tritylpyrazole (6a) was deprotected with aqueous $\mathrm{HCl}$ to give 18, which was then treated with trityl chloride or benzyl bromide under basic conditions. An $E / Z$ mixture of 4-allyloxy-3-(1-propenyl)-1H-1-tritylpyrazole (19a) was obtained exclusively owing to the steric repulsion between the propenyl group on the pyrazole ring and an introduced bulky trityl group. However, $N$-benzylation of 18 afforded a mixture of $19 b$ and $6 \mathbf{b}$ in a ca. 4:1 ratio in $60 \%$ combined yield, and separation gave pure $19 \mathbf{b}$ in $25 \%$ yield. The obtained substrates $19 \mathbf{a}$ and $\mathbf{1 9 b}$ were 
independently treated with $5 \mathrm{~mol} \% \mathrm{Grubbs}^{2 \mathrm{nd}}$ at rt to afford the desired RCM products 20a and 20b, respectively, in good yields.

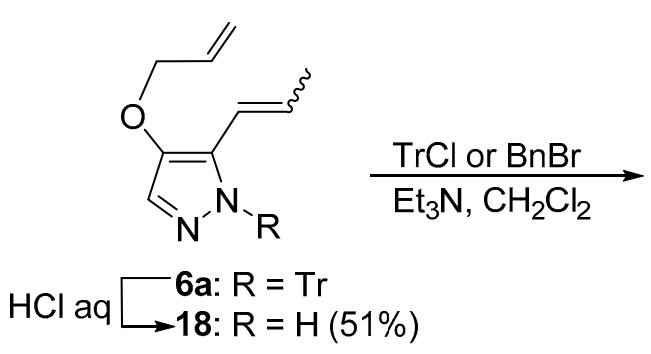

Scheme 3. Synthesis of 2,5-dihydropyrano[3,2-c]pyrazoles (20).

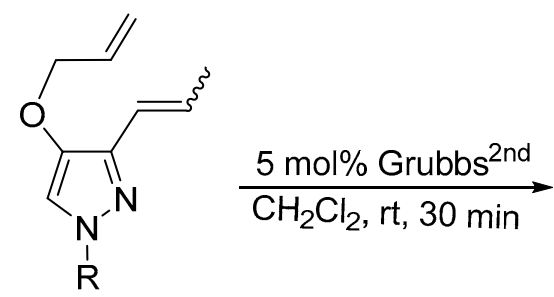

19a: $R=\operatorname{Tr}(69 \%)$

19b: $R=B n(25 \%)$

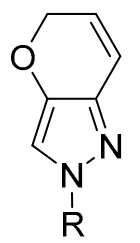

20a: $R=\operatorname{Tr}(76 \%)$

20b: $R=\operatorname{Bn}(74 \%)$

\section{Conclusions}

We synthesized 1,5-, 1,7-, and 2,5-dihydropyrano[3,2-c]pyrazoles (7, 8, and 20) from 5-allyl-4-hydroxy-1H-1-tritylpyrazoles via a combination of the Claisen rearrangement, ruthenium-hydride-catalyzed double bond isomerization, $O$-alkenylation, and RCM. In the synthesis of 1,5-dihydropyrano[3,2-c]pyrazoles 7, the presence of a substituent on the 5-alkenyl group inhibited smooth RCM through steric hindrance. In these cases, MW-aided reactions were effective, but gave various products. Towards the selective synthesis of 1,7-dihydro-1-tritylpyrano[3,2-c]pyrazole 8a, temperature-dependent selective dehydrobromination was effective for preparing the RCM substrate 13b. For the synthesis of 2,5-dihydropyrano[3,2-c]pyrazoles 20, a deprotection-reprotection sequence was applied to obtain the RCM substrate 19.

\section{Materials and Methods}

Infrared (IR) spectra were obtained using a Perkin Elmer 1720X FT-IR spectrometer (Perkin Elmer, Wattham, MA, USA). HRMS was performed using a JEOL JMS-700 (2) mass spectrometer (JEOL, Tokyo, Japan). NMR spectra were recorded at $27^{\circ} \mathrm{C}$ using Agilent 300, 400-MR-DD2, and 600-DD2 spectrometers in $\mathrm{CDCl}_{3}$ using tetramethylsilane (TMS) as the internal standard. Liquid column chromatography was conducted using silica gel BW127ZH (Fuji Silysia Chemical Ltd., Tokyo, Japn). Analytical and preparative thin layer chromatography (TLC) analyses were performed using pre-coated Merck glass plates (silica gel $60 \mathrm{~F}_{254}$ ), and the compounds were visualized by dipping the plates in an ethanol solution of phosphomolybdic acid followed by heating (Merk \& Co., Inc., Darmstadt, Germany). MW-assisted reactions were carried out using a Biotage Initiator ${ }^{\circledR}$ (Basel, Switzerland). Anhydrous $\mathrm{CH}_{2} \mathrm{CH}_{2}$ was purchased from Wako Pure Chemical Industries (Osaka, Japan).

\subsection{Synthesis of (E)-Methyl 4-((1-Benzyl-1H-pyrazol-4-yl)oxy)but-2-enoate (1e)}

To 1-benzyl-4-formyl-1H-pyrazole $(200 \mathrm{mg}, 1.07 \mathrm{mmol})$ in $\mathrm{CH}_{2} \mathrm{Cl}_{2}(5 \mathrm{~mL})$ was added $70 \%$ meta-chloroperoxybenzoic acid $(397.6 \mathrm{mg}, 1.61 \mathrm{mmol})$ at $0{ }^{\circ} \mathrm{C}$. After it was stirred overnight at room temperature, the mixture was quenched by adding aqueous $\mathrm{NaHCO}_{3}$ and then extracted with $\mathrm{CH}_{2} \mathrm{Cl}_{2}$. The organic layer was dried over $\mathrm{MgSO}_{4}$, filtered, and evaporated to give a crude residue. The crude material was dissolved in $t-\mathrm{BuOH}-\mathrm{CH}_{2} \mathrm{Cl}_{2}(5 \mathrm{~mL} / 5 \mathrm{~mL})$ at $40{ }^{\circ} \mathrm{C}$, and then potassium tert-butoxide (428.6 mg, $3.82 \mathrm{mmol}$ ) was added to the solution. After it was stirred overnight at $40{ }^{\circ} \mathrm{C}$, the mixture was quenched with saturated aqueous $\mathrm{NH}_{4} \mathrm{Cl}$ and extracted with $\mathrm{CH}_{2} \mathrm{Cl}_{2}$. The separated organic layer was dried over $\mathrm{MgSO}_{4}$, filtered, and evaporated under reduced pressure to afford a crude residue, which was purified using silica gel column chromatography (eluent: EtOAc:hexane $=1: 3$ ) to afford (E/Z)-1e (128.1 mg, 44\%): oil; IR (film) $v_{\max } 1724$ (C=O), 1574 (C=C), 1437 (C=C) cm ${ }^{-1},{ }^{1} \mathrm{H}$ NMR (400 $\left.\mathrm{MHz}, \mathrm{CDCl}_{3}\right): \delta 3.74(3 \mathrm{H}, \mathrm{s},-\mathrm{COOMe}), 4.52\left(2 \mathrm{H}, \mathrm{dd}, J=4.1,1.9 \mathrm{~Hz},-\mathrm{OCH}_{2} \mathrm{CH}=\mathrm{CH}-\right), 6.13(1 \mathrm{H}, \mathrm{br} \mathrm{d}, J=$ $15.9 \mathrm{~Hz},-\mathrm{COCH}=\mathrm{CH}-), 6.99\left(1 \mathrm{H}, \mathrm{dt}, J=15.9,4.1 \mathrm{~Hz},-\mathrm{CH}_{2} \mathrm{CH}=\mathrm{CH}-\right), 7.05(1 \mathrm{H}, \mathrm{d}, J=0.6 \mathrm{~Hz}$, pyrazole-H), $7.18(2 \mathrm{H}, \mathrm{br} \mathrm{d}, J=8.0 \mathrm{~Hz}, \mathrm{Bn}-\mathrm{H}), 7.30-7.35\left(4 \mathrm{H}, \mathrm{m}, \mathrm{Bn}-\mathrm{H}\right.$, pyrazole-H); ${ }^{13} \mathrm{C}$ NMR $\left(100 \mathrm{MHz}, \mathrm{CDCl}_{3}\right): \delta$ 
51.6, 56.6, 70.0, 115.1, 121.4, 127.2, 127.5, 128.0, 128.7, 136.3, 142.5, 145.2, 166.4; high-resolution electron ionization mass spectrometry (HREIMS) $m / z$ calcd. for $\mathrm{C}_{15} \mathrm{H}_{16} \mathrm{~N}_{2} \mathrm{O}_{3}\left(\mathrm{M}^{+}\right)$272.1161, found 272.1163.

*(E)-Methyl 4-((1-trityl-1H-pyrazol-4-yl)oxy)but-2-enoate (1f) was synthesized in a similar manner as 1e, but it was not rearranged under the thermal condition described below. 1f: colorless crystals $\left(\mathrm{CH}_{2} \mathrm{Cl}_{2}\right)$; mp 155-158 ${ }^{\circ} \mathrm{C}$; IR (film) $v_{\max } 1725(\mathrm{C}=\mathrm{O}), 1572(\mathrm{C}=\mathrm{C}), 1492(\mathrm{C}=\mathrm{C}), 1442(\mathrm{C}=\mathrm{C}) \mathrm{cm}^{-1} ;{ }^{1} \mathrm{H}$ NMR $\left(400 \mathrm{MHz}, \mathrm{CDCl}_{3}\right): \delta 3.76(3 \mathrm{H}, \mathrm{s},-\mathrm{COOMe}), 4.53\left(2 \mathrm{H}, \mathrm{dd}, J=4.1,2.0 \mathrm{~Hz},-\mathrm{OCH}_{2} \mathrm{CH}=\mathrm{CH}-\right)$, $5.20\left(2 \mathrm{H}, \mathrm{s}, \mathrm{ArCH} \mathrm{CH}_{2} \mathrm{Ph}\right), 6.14(1 \mathrm{H}, \mathrm{dt}, J=15.9,1.9 \mathrm{~Hz},-\mathrm{COCH}=\mathrm{CH}-), 7.00(1 \mathrm{H}, \mathrm{dt}, J=15.9,4.1 \mathrm{~Hz}$, $\left.-\mathrm{CH}_{2} \mathrm{CH}=\mathrm{CH}-\right), 7.05(1 \mathrm{H}, \mathrm{s}$, pyrazole-H), 7.13-7.18 (6H, m, Tr-H), 7.30-7.35 (9H, m, Tr-H), $7.42(1 \mathrm{H}, \mathrm{s}$, pyrazole-H); ${ }^{13} \mathrm{C}$ NMR $\left(100 \mathrm{MHz}, \mathrm{CDCl}_{3}\right): \delta 51.7,70.0,78.7,118.4,121.5,127.68,127.71,127.9,130.1$, 142.5, 143.0, 143.8, 166.4; HREIMS $m / z$ calcd. for $\mathrm{C}_{27} \mathrm{H}_{24} \mathrm{~N}_{2} \mathrm{O}_{3}\left(\mathrm{M}^{+}\right) 424.1786$, found 424.1779.

\subsection{Synthesis of Methyl 2-(1-Benzyl-4-hydroxy-1H-pyrazol-5-yl)but-3-enoate (2e)}

A sealed microwave vial containing a solution of 1 e $(128.1 \mathrm{mg}, 0.47 \mathrm{mmol})$ in 1,2-dimethoxyethane (DME) $(2 \mathrm{~mL})$ was heated under microwave irradiation at $200^{\circ} \mathrm{C}$ for $30 \mathrm{~min}$. After it had cooled, the reaction mixture was quenched with saturated aqueous $\mathrm{NH}_{4} \mathrm{Cl}$ and extracted with $\mathrm{CH}_{2} \mathrm{Cl}_{2}$. The separated organic layer was dried over $\mathrm{MgSO}_{4}$, filtered, and evaporated under reduced pressure to afford a crude residue, which was purified using silica gel column chromatography (eluent: EtOAc:hexane $=1: 1)$ to afford $2 \mathbf{e}$ with a small amount of the isomer, $5 \mathbf{e}(53.9 \mathrm{mg}, 42 \%)$.

2e (major) and 5e (minor) in ca. 2:1 ratio: oil; IR (film) $v_{\max } 1716(\mathrm{C}=\mathrm{O}), 1497(\mathrm{C}=\mathrm{C}), 1435$ $(\mathrm{C}=\mathrm{C}) \mathrm{cm}^{-1} ;{ }^{1} \mathrm{H}$ NMR $\left(400 \mathrm{MHz}, \mathrm{CDCl}_{3}\right): \delta 1.65\left(1 \mathrm{H}, \mathrm{d}, J=7.3 \mathrm{~Hz},=\mathrm{CHCH}_{3}\right.$ of $\left.5 \mathbf{e}\right), 3.64(1 \mathrm{H}, \mathrm{s}$, -COOMe of 5e), $3.68(2 \mathrm{H}, \mathrm{s},-\mathrm{COOMe}$ of $2 \mathbf{e}), 4.37(0.7 \mathrm{H}, \mathrm{br} \mathrm{d}, J=7.0 \mathrm{~Hz}, \mathrm{ArCH}(\mathrm{COOMe}) \mathrm{CH}=), 4.93$ $(0.7 \mathrm{H}, \mathrm{dd}, J=17.0,1.5 \mathrm{~Hz},-\mathrm{CH}=\mathrm{CHH}), 5.01\left(0.6 \mathrm{H}, \mathrm{s}, \mathrm{ArCH}_{2} \mathrm{Ph}\right), 5.12(0.7 \mathrm{H}, \mathrm{dd}, J=10.3,1.5 \mathrm{~Hz}$, $-\mathrm{CH}=\mathrm{CHH}$ of $\mathbf{2 e}), 5.19(0.7 \mathrm{H}, \mathrm{brd}, J=16.1 \mathrm{~Hz}, \mathrm{ArCHHPh}$ of $2 \mathbf{e}), 5.25(0.7 \mathrm{H}, \mathrm{br} \mathrm{d}, J=16.1 \mathrm{~Hz}, \mathrm{ArCH} H \mathrm{Ph}$ of $2 \mathbf{e}), 5.86\left(1 \mathrm{H}, \mathrm{ddd}, J=17.0,10.3,6.5 \mathrm{~Hz},-\mathrm{CH}(\mathrm{COOMe}) \mathrm{CH}=\mathrm{CH}_{2}\right.$ of $\left.2 \mathbf{e}\right), 6.83(0.6 \mathrm{H}, \mathrm{br}$ s, $-\mathrm{OH}$ of 2e), 7.03-7.05 (2H, m, Ph-H), 7.19-7.31 (3H, m, Ph-H; 0.3H, m, overlapped, $=\mathrm{CHCH}_{3}$ of $\left.5 \mathbf{e}\right), 7.30(1 \mathrm{H}$, br s, pyrazole-H); ${ }^{13} \mathrm{C} \mathrm{NMR}\left(150 \mathrm{MHz}, \mathrm{CDCl}_{3}\right): \delta 15.8$ (5e), 46.4, 52.3 (5e), 53.2, 54.4 (5e), 54.6, 118.7, 120.6, 122.4 (5e), 122.9 (5e), 126.6, 127.2 (5e), 127.6 (5e), 127.9, 128.2 (5e), 128.40 (5e), 128.44, 129.1, 130.7, 136.9 (5e), 139.8 (5e), 140.9, 147.0, 166.5 (5e), 173.2; HREIMS $m / z$ calcd. for $\mathrm{C}_{15} \mathrm{H}_{16} \mathrm{~N}_{2} \mathrm{O}_{3}\left(\mathrm{M}^{+}\right)$272.1161, found 272.1162 .

\subsection{Double Bond Migration of 5-Allyl-4-hydroxy-1H-pyrazoles (Scheme 1)}

General procedure: To a toluene solution (10 mL) of 5-allyl-4-hydroxy-1-trityl-1H-pyrazole (2a) $(0.434 \mathrm{~g}, 1.19 \mathrm{mmol})$ in a microwave vial $(5-20 \mathrm{~mL}), \mathrm{RuClH}(\mathrm{CO})\left(\mathrm{PPh}_{3}\right)_{3}(56.6 \mathrm{mg}, 0.059 \mathrm{mmol})$ was added. The reaction vial was sealed and then heated at $150{ }^{\circ} \mathrm{C}$ for $15 \mathrm{~min}$ under microwave irradiation. The cooled reaction mixture was evaporated to give a crude residue, which was purified using column chromatography (eluent: hexane:EtOAc $=1: 1)$ to afford 4-hydroxy-5-(1-propenyl)-1-trityl-1H-pyrazole (5a) $(0.323 \mathrm{~g}, 74 \%$ yield $)$ as an $E / Z$ mixture.

**Pure starting material gave the desired product as described above, but a small contamination inhibited the isomerization. In that case, a toluene-MeOH (9:1) solvent system was effective for isolating the desired product.

5a: oil; IR (film) $v_{\max } 3268(-\mathrm{OH}), 1597(\mathrm{C}=\mathrm{C}), 1494(\mathrm{C}=\mathrm{C}), 1446(\mathrm{C}=\mathrm{C}) \mathrm{cm}^{-1} ;{ }^{1} \mathrm{H}$ NMR $(400 \mathrm{MHz}$, $\left.\mathrm{CDCl}_{3}\right): \delta 1.43\left(3 \mathrm{H}, \mathrm{dd}, J=6.5,1.2 \mathrm{~Hz},=\mathrm{CHCH}_{3}\right), 5.17(1 \mathrm{H}, \mathrm{dd}, J=11.4,1.4 \mathrm{~Hz}, \mathrm{ArCH}=\mathrm{CH}-), 5.23(1 \mathrm{H}$, $\left.\mathrm{dq}, J=11.4,6.6 \mathrm{~Hz},-\mathrm{CH}=\mathrm{CHCH}_{3}\right), 7.09-7.34\left(16 \mathrm{H}, \mathrm{m}, \mathrm{Tr}-\mathrm{H}\right.$, pyrazole-H); ${ }^{13} \mathrm{C}$ NMR $\left(100 \mathrm{MHz}, \mathrm{CDCl}_{3}\right)$ : $\delta 14.9,78.8,118.3,126.2,127.3,127.4,127.6,127.8,129.6,130.0,130.1,130.28,130.34,142.6$; HREIMS m/z calcd. for $\mathrm{C}_{25} \mathrm{H}_{22} \mathrm{~N}_{2} \mathrm{O}\left(\mathrm{M}^{+}\right) 366.1732$, found 366.1731.

$(E / Z)-1-B e n z y l-4-h y d r o x y-5-(1-p r o p e n y l)-1 H$-pyrazole $(5 \mathbf{b}): E / Z$ mixture in ca. 5:1 ratio $(X)$; oil; IR (film) $v_{\max } 3031$ (-OH), 1589 (C=C), 1496 (C=C), $1454(\mathrm{C}=\mathrm{C}) \mathrm{cm}^{-1} ;{ }^{1} \mathrm{H}$ NMR $\left(600 \mathrm{MHz}, \mathrm{CDCl}_{3}\right): \delta$ $1.72\left(0.5 \mathrm{H}, \mathrm{dd}, J=6.8,1.5 \mathrm{~Hz},-\mathrm{CH}=\mathrm{CHCH}_{3}\right.$ of $(E)$-isomer $), 1.83\left(2.5 \mathrm{H}, \mathrm{dd}, J=6.8,1.8 \mathrm{~Hz},-\mathrm{CH}=\mathrm{CHCH}_{3}\right.$ of (Z)-isomer), $5.15\left(0.3 \mathrm{H}, \mathrm{s},-\mathrm{NCH}_{2} \mathrm{Ph}\right.$ of $(\mathrm{Z})$-isomer $), 5.24\left(1.5 \mathrm{H}, \mathrm{s},-\mathrm{NCH}_{2} \mathrm{Ph}\right.$ of $(E)$-isomer), $5.93(0.15 \mathrm{H}$, $\mathrm{dq}, J=10.1,6.8 \mathrm{~Hz},-\mathrm{CH}=\mathrm{CHCH}_{3}$ of $(\mathrm{Z})$-isomer $), 5.99\left(0.15 \mathrm{H}, \mathrm{dq}, J=10.1,1.5 \mathrm{~Hz}, \mathrm{ArCH}=\mathrm{CHCH}_{3}\right.$ of 
(Z)-isomer), $6.15\left(0.85 \mathrm{H}, \mathrm{dq}, J=16.1,1.5 \mathrm{~Hz}, \mathrm{ArCH}=\mathrm{CHCH}_{3}\right.$ of $(E)$-isomer $), 6.38(0.85 \mathrm{H}, \mathrm{dq}, J=16.1$, $6.8 \mathrm{~Hz},-\mathrm{CH}=\mathrm{CHCH}_{3}$ of $(E)$-isomer), 7.06-7.09 $(6 \mathrm{H}, \mathrm{m}, \mathrm{Tr}-\mathrm{H}), 7.16(1 \mathrm{H}, \mathrm{s}$, pyrazole- $\mathrm{H}), 7.22-7.31(9 \mathrm{H}, \mathrm{m}$, $\mathrm{Tr}-\mathrm{H}) ;{ }^{13} \mathrm{C}$ NMR of $(E)$-isomer $\left(150 \mathrm{MHz}, \mathrm{CDCl}_{3}\right): \delta 19.2,53.7,116.7,126.6,127.6,127.9,128.7,130.3$, 137.1, 138.9 (two carbon signals were deduced to have overlapped); (Z)-isomer: $\delta 15.4,54.0,115.4$, $126.8,126.9,127.9,128.6,133.4,137.0,138.4$ (two carbon signals were deduced to have overlapped); HREIMS $m / z$ calcd. for $\mathrm{C}_{13} \mathrm{H}_{14} \mathrm{~N}_{2} \mathrm{O}\left(\mathrm{M}^{+}\right)$214.1106, found 214.1104 .

(E/Z)-1-Benzyl-4-hydroxy-5-(1-(1-methyl)propen-1-yl)-1H-pyrazole (5c): $E / Z$ ratio = ca. 1:1; oil; IR (film) $v_{\max } 3063(\mathrm{OH}), 1563(\mathrm{C}=\mathrm{C}), 1497(\mathrm{C}=\mathrm{C}), 1456(\mathrm{C}=\mathrm{C}) \mathrm{cm}^{-1} ;{ }^{1} \mathrm{H}$ NMR of $E / Z$ mixture $(400 \mathrm{MHz}$, $\left.\mathrm{CDCl}_{3}\right): \delta 1.41\left(1.6 \mathrm{H}, \mathrm{dd}, J=6.9,1.5 \mathrm{~Hz},-\mathrm{CH}=\mathrm{CHCH}_{3}\right), 1.73\left(1.4 \mathrm{H}, \mathrm{dd}, J=6.8,1.2 \mathrm{~Hz},-\mathrm{CH}=\mathrm{CHCH}_{3}\right)$, $1.79\left(3 \mathrm{H}\right.$, br s, $\left.\mathrm{C}_{\mathrm{q}} \mathrm{CH}_{3}\right), 4.30(0.47 \mathrm{H}$, br s, $-\mathrm{OH}), 4.43(0.53 \mathrm{H}, \mathrm{br} \mathrm{s},-\mathrm{OH}), 5.08\left(0.9 \mathrm{H}, \mathrm{s},-\mathrm{NCH}_{2} \mathrm{Ph}\right), 5.15$ $\left(1.1 \mathrm{H}, \mathrm{s},-\mathrm{NCH}_{2} \mathrm{Ph}\right), 5.57\left(0.47 \mathrm{H}, \mathrm{qq}, J=6.8,1.6 \mathrm{~Hz},-\mathrm{CCH}=\mathrm{CHCH}_{3}\right), 5.78(0.53 \mathrm{H}, \mathrm{qq}, J=6.9,1.6 \mathrm{~Hz}$, $\left.-\mathrm{CCH}_{3}=\mathrm{CHCH}_{3}\right), 7.03(0.94 \mathrm{H}$, br d, $J=6.7 \mathrm{~Hz}, \mathrm{Ph}-\mathrm{H}), 7.07(1.06 \mathrm{H}$, br d, $J=6.7 \mathrm{~Hz}, \mathrm{Ph}-\mathrm{H}), 7.06-7.09$ (6H, m, Ph-H), 7.16-7.36 (3H, m, Ph-H), 7.21 (1H, s, pyrazole-H); ${ }^{13} \mathrm{C}$ NMR of $E$ / Z mixture (100 MHz, $\mathrm{CDCl}_{3}$ ): $\delta$ 14.0, 15.0, 16.2, 32.0, 53.9, 54.1, 124.2, 124.6, 126.8, 127.2, 127.4, 127.5, 127.91, 127.94, 128.46, $128.49,128.6,129.8,130.0,132.6,137.3,137.6,137.8$; HREIMS $m / z$ calcd. for $\mathrm{C}_{14} \mathrm{H}_{16} \mathrm{~N}_{2} \mathrm{O}\left(\mathrm{M}^{+}\right)$228.1263, found 228.1260 .

(E/Z)-1-Benzyl-4-hydroxy-5-(1-(1-phenyl)propenyl)-1H-pyrazole (5d): isomer ratio = ca. 5:1; oil; IR (film) $v_{\max } 3031(\mathrm{OH}), 1573(\mathrm{C}=\mathrm{C}), 1496(\mathrm{C}=\mathrm{C}) \mathrm{cm}^{-1} ;{ }^{1} \mathrm{H}$ NMR $\left(100 \mathrm{MHz}, \mathrm{CDCl}_{3}\right): \delta 1.55(2.5 \mathrm{H}, \mathrm{d}$, $\left.J=7.0 \mathrm{~Hz},=\mathrm{CHCH}_{3}\right), 1.85\left(0.5 \mathrm{H}, \mathrm{d}, J=7.3 \mathrm{~Hz},=\mathrm{CHCH}_{3}\right), 4.61(1 \mathrm{H}, \mathrm{br} \mathrm{s}, J=14.8 \mathrm{~Hz},-\mathrm{NCHHPh}), 4.95$ $(1 \mathrm{H}, \mathrm{br} \mathrm{s}, J=15.3 \mathrm{~Hz},-\mathrm{NCHHPh}), 5.94\left(0.16 \mathrm{H}, \mathrm{q}, J=7.2 \mathrm{~Hz}, \mathrm{C}_{\mathrm{q}}=\mathrm{CHCH}_{3}\right), 6.31(0.84 \mathrm{H}, \mathrm{br} \mathrm{q}, J=7.0 \mathrm{~Hz}$, $\left.\mathrm{C}_{\mathrm{q}}=\mathrm{CHCH}_{3}\right), 6.87-6.90(0.66 \mathrm{H}, \mathrm{m}, \mathrm{Ph}-\mathrm{H}), 6.93-6.96(1.34 \mathrm{H}, \mathrm{m}, \mathrm{Ph}-\mathrm{H}), 7.06-7.34(4 \mathrm{H}, \mathrm{m}, \mathrm{Ph}-\mathrm{H}), 7.32(1 \mathrm{H}$, s, pyrazole-H); ${ }^{13} \mathrm{C}$ NMR $\left(100 \mathrm{MHz}, \mathrm{CDCl}_{3}\right): \delta 15.7,54.3,126.2,127.0,127.38,127.45,127.6,128.3,128.4$, 128.6, 128.9, 129.3, 131.1, 136.9, 139.9 (minor isomer: 15.4, 54.0, 126.4, 127.68, 127.8); HREIMS $\mathrm{m} / \mathrm{z}$ calcd. for $\mathrm{C}_{19} \mathrm{H}_{18} \mathrm{~N}_{2} \mathrm{O}\left(\mathrm{M}^{+}\right) 290.1419$, found 290.1417 .

(E/Z)-1-Benzyl-4-hydroxy-5-(1-(1-methoxycarbonyl)propenyl)-1H-pyrazole (5e): $E / Z$ mixture in ca. 13:1 ratio; oil; IR (film) $v_{\max } 3090(\mathrm{OH}), 1716(\mathrm{C}=\mathrm{O}), 1507(\mathrm{C}=\mathrm{C}), 1436(\mathrm{C}=\mathrm{C}) \mathrm{cm}^{-1} ;{ }^{1} \mathrm{H}$ NMR $\left(400 \mathrm{MHz}, \mathrm{CDCl}_{3}\right): \delta 1.72\left(2.6 \mathrm{H}, \mathrm{d}, J=7.3 \mathrm{~Hz},-\mathrm{CH}=\mathrm{CHCH}_{3}\right.$ of major isomer), $1.83(0.4 \mathrm{H}, \mathrm{d}, J=7.2 \mathrm{~Hz}$, $-\mathrm{CH}=\mathrm{CHCH}_{3}$ of minor isomer), $3.66\left(2.6 \mathrm{H}, \mathrm{s},-\mathrm{OCH}_{3}\right.$ of major isomer $), 3.71\left(0.4 \mathrm{H}, \mathrm{s},-\mathrm{OCH}_{3}\right.$ of minor isomer), $4.73(1 \mathrm{H}, \mathrm{s},-\mathrm{OH}), 5.06\left(1.86 \mathrm{H}, \mathrm{s},-\mathrm{NCH}_{2} \mathrm{Ph}\right.$ of major isomer), $5.17\left(0.14 \mathrm{H}, \mathrm{s},-\mathrm{NCH}_{2} \mathrm{Ph}\right.$ of minor isomer), $7.04(2 \mathrm{H}, \mathrm{br} \mathrm{d}, J=6.6 \mathrm{~Hz}, \mathrm{Ph}-\mathrm{H}), 7.19-7.30\left(4 \mathrm{H}, \mathrm{m}, \mathrm{Ph}-\mathrm{H},=\mathrm{CHCH}_{3}\right), 7.33(1 \mathrm{H}, \mathrm{s}$, pyrazole-H); ${ }^{13} \mathrm{C}$ NMR $\left(100 \mathrm{MHz}, \mathrm{CDCl}_{3}\right): \delta 15.8,52.3,54.5,122.2,122.9,127.3,127.6,128.1,128.4,136.7,140.0,147.5$, 166.5; HREIMS $m / z$ calcd. for $\mathrm{C}_{15} \mathrm{H}_{16} \mathrm{~N}_{2} \mathrm{O}_{3}\left(\mathrm{M}^{+}\right)$272.1161, found 272.1160.

\subsection{O-Allylation of 1-Protected 5- or 3-Allyl-4-allyloxy-1H-pyrazoles (Scheme 1)}

General procedure: To a solution of an $E / Z$ mixture of 4-hydroxy-5-(1-propenyl)- $1 H$ 1-tritylpyrazole (5a) $(0.410 \mathrm{~g}, 1.12 \mathrm{mmol})$ in acetone $(2 \mathrm{~mL}), 20 \%$ aqueous $\mathrm{NaOH}(1 \mathrm{~mL})$ and allyl bromide $(142 \mu \mathrm{L}, 1.68 \mathrm{mmol})$ were added. The reaction mixture was stirred for $1 \mathrm{~h}$ and then quenched with saturated aqueous $\mathrm{NH}_{4} \mathrm{Cl}$ and extracted with $\mathrm{CH}_{2} \mathrm{Cl}_{2}$. The organic layer was dried over anhydrous $\mathrm{MgSO}_{4}$, filtered, and evaporated. The crude residue was purified with column chromatography (eluent: hexane:EtOAc $=3: 1$ ) to afford 4-allyloxy-5-(1-propenyl)-1H-1-tritylpyrazole (6a) ( $E / Z$ mixture in ca. 3:1 ratio, $0.334 \mathrm{~g}$, 73\% yield).

(E)-6a: $\mathrm{mp}$ 152-155 ${ }^{\circ} \mathrm{C}$; IR (KBr) $v_{\max } 1567(\mathrm{C}=\mathrm{C}), 1491(\mathrm{C}=\mathrm{C}), 1446(\mathrm{C}=\mathrm{C}) \mathrm{cm}^{-1} ;{ }^{1} \mathrm{H}$ NMR $(400$ $\left.\mathrm{MHz}, \mathrm{CDCl}_{3}\right): \delta 1.40\left(3 \mathrm{H}, \mathrm{dd}, J=6.7,1.5 \mathrm{~Hz}, \mathrm{CH}_{3} \mathrm{CH}=\right), 4.50\left(2 \mathrm{H}, \mathrm{dt}, J=5.3,1.5 \mathrm{~Hz},-\mathrm{OCH}_{2} \mathrm{CH}=\mathrm{CH}_{2}\right)$, $5.26\left(1 \mathrm{H}, \mathrm{dq}, J=15.8,1.4 \mathrm{~Hz},-\mathrm{CH}_{2} \mathrm{CH}=\mathrm{CHH}\right), 5.37\left(1 \mathrm{H}, \mathrm{dq}, J=17.2,1.5 \mathrm{~Hz},-\mathrm{CH}_{2} \mathrm{CH}=\mathrm{CHH}\right), 5.46$ $\left(1 \mathrm{H}, \mathrm{dq}, J=15.8,1.4 \mathrm{~Hz}, \mathrm{ArCH}=\mathrm{CHCH}_{3}\right), 6.04\left(1 \mathrm{H}, \mathrm{ddt}, J=17.2,10.5,5.3 \mathrm{~Hz},-\mathrm{OCH}_{2} \mathrm{CH}=\mathrm{CH}_{2}\right), 6.14$ $\left(1 \mathrm{H}, \mathrm{dq}, J=15.8,6.7 \mathrm{~Hz}, \mathrm{ArCH}=\mathrm{CHCH}_{3}\right), 7.07-7.16(6 \mathrm{H}, \mathrm{m}, \mathrm{Tr}-\mathrm{H}), 7.24-7.39(9 \mathrm{H}, \mathrm{m}, \mathrm{Tr}-\mathrm{H}), 7.32(1 \mathrm{H}$, s, pyrazole-H); ${ }^{13} \mathrm{C}$ NMR $\left(100 \mathrm{MHz}, \mathrm{CDCl}_{3}\right): \delta 18.9,72.0,79.0,117.5,120.0,124.7,127.3,127.4,127.6$, 128.5, 130.3, 133.5, 142.9, 143.5; HREIMS $m / z$ calcd. for $\mathrm{C}_{28} \mathrm{H}_{26} \mathrm{~N}_{2} \mathrm{O}\left(\mathrm{M}^{+}\right)$406.2055, found 406.2047.

(Z)-6a: $\mathrm{mp} 82-86{ }^{\circ} \mathrm{C}$; IR (KBr) $v_{\max } 1567(\mathrm{C}=\mathrm{C}), 1491$ (C=C), $1446(\mathrm{C}=\mathrm{C}) \mathrm{cm}^{-1} ;{ }^{1} \mathrm{H}$ NMR $(400 \mathrm{MHz}$, $\left.\mathrm{CDCl}_{3}\right): \delta 1.42\left(3 \mathrm{H}, \mathrm{d}, J=5.1 \mathrm{~Hz}, \mathrm{CH}_{3} \mathrm{CH}=\right), 4.47\left(2 \mathrm{H}, \mathrm{dt}, J=5.5,1.6 \mathrm{~Hz},-\mathrm{OCH}_{2} \mathrm{CH}^{-} \mathrm{CH}_{2}\right), 5.16-5.20$ 
$(2 \mathrm{H}, \mathrm{m}), 5.22\left(1 \mathrm{H}, \mathrm{dq}, J=10.6,1.4 \mathrm{~Hz},-\mathrm{CH}_{2} \mathrm{CH}=\mathrm{CHH}\right), 5.34\left(1 \mathrm{H}, \mathrm{dq}, J=17.2,1.5 \mathrm{~Hz},-\mathrm{CH}_{2} \mathrm{CH}=\mathrm{CHH}\right)$, $6.00\left(1 \mathrm{H}, \mathrm{ddt}, J=17.2,10.6,5.6 \mathrm{~Hz},-\mathrm{OCH}_{2} \mathrm{CH}=\mathrm{CH}_{2}\right), 7.03-7.17(6 \mathrm{H}, \mathrm{m}, \mathrm{Tr}-\mathrm{H}), 7.21-7.32(9 \mathrm{H}, \mathrm{m}, \mathrm{Tr}-\mathrm{H})$, $7.37\left(1 \mathrm{H}\right.$, s, pyrazole-H); ${ }^{13} \mathrm{C}$ NMR $\left(100 \mathrm{MHz}, \mathrm{CDCl}_{3}\right): \delta 15.4,72.0,78.9,117.4,117.9,124.9,127.2$, 127.3, 127.9, 129.8, 130.1, 133.7, 142.6, 142.9; HREIMS $m / z$ calcd. for $\mathrm{C}_{28} \mathrm{H}_{26} \mathrm{~N}_{2} \mathrm{O}\left(\mathrm{M}^{+}\right)$406.2046, found 406.2050.

(E/Z)-4-Allyloxy-1-benzyl-5-(1-propenyl)-1H-pyrazole (6b) (an inseparable $E / Z$ mixture in a ca. 8:2 ratio, $0.334 \mathrm{~g}$, 73\% yield): oil; IR (film) $v_{\max } 1566(\mathrm{C}=\mathrm{C}), 1495(\mathrm{C}=\mathrm{C}), 1452(\mathrm{C}=\mathrm{C}) \mathrm{cm}^{-1}$; HREIMS $m / z$ calcd. for $\mathrm{C}_{16} \mathrm{H}_{18} \mathrm{~N}_{2} \mathrm{O}\left(\mathrm{M}^{+}\right)$254.1419, found 254.1421. (E)-isomer: ${ }^{1} \mathrm{H}$ NMR $\left(600 \mathrm{MHz}, \mathrm{CDCl}_{3}\right): \delta$ $1.82\left(3 \mathrm{H}, \mathrm{d}, J=6.7,1.8 \mathrm{~Hz}, \mathrm{CH}_{3} \mathrm{CH}=\right), 4.50\left(2 \mathrm{H}, \mathrm{dt}, J=5.4,1.4 \mathrm{~Hz},-\mathrm{OCH}_{2} \mathrm{CH}=\mathrm{CH}_{2}\right), 5.26(1 \mathrm{H}, \mathrm{dq}, J=$ $\left.10.6,1.4 \mathrm{~Hz},-\mathrm{CH}_{2} \mathrm{CH}=\mathrm{CHH}\right), 5.28\left(2 \mathrm{H}, \mathrm{s}, \mathrm{NCH}_{2} \mathrm{Ph}\right), 5.39\left(1 \mathrm{H}, \mathrm{ddd}, J=17.3,3.2,1.8 \mathrm{~Hz},-\mathrm{CH}_{2} \mathrm{CH}=\mathrm{CHH}\right)$, $6.05\left(1 \mathrm{H}, \mathrm{ddt}, J=17.3,10.6,5.4 \mathrm{~Hz},-\mathrm{OCH}_{2} \mathrm{CH}=\mathrm{CH}_{2}\right), 6.16\left(1 \mathrm{H}, \mathrm{br} \mathrm{d}, J=15.8 \mathrm{~Hz}, \mathrm{ArCH}=\mathrm{CHCH}_{3}\right)$, $6.46\left(1 \mathrm{H}, \mathrm{dq}, J=15.8,6.7 \mathrm{~Hz},-\mathrm{CH}=\mathrm{CHCH}_{3}\right), 7.07(2 \mathrm{H}, \mathrm{br} \mathrm{d}, J=7.6 \mathrm{~Hz}, \mathrm{Ph}-\mathrm{H}), 7.24(1 \mathrm{H}, \mathrm{br} \mathrm{t}, J=7.6$ $\mathrm{Hz}, \mathrm{Ph}-\mathrm{H}), 7.26\left(1 \mathrm{H}, \mathrm{s}\right.$, pyrazole-H), $7.30(2 \mathrm{H}, \mathrm{br} \mathrm{t}, J=7.6 \mathrm{~Hz}, \mathrm{Ph}-\mathrm{H}) ;{ }^{13} \mathrm{C}$ NMR $\left(150 \mathrm{MHz}, \mathrm{CDCl}_{3}\right)$ : $\delta 19.3,53.9,72.2,116.6,117.5,125.8,126.5,126.8,127.5,128.6,128.7,129.6,133.5,137.2 ;(Z)$-isomer: ${ }^{1} \mathrm{H}$ NMR $\left(600 \mathrm{MHz}, \mathrm{CDCl}_{3}\right): \delta 1.72\left(3 \mathrm{H}, \mathrm{dd}, J=6.8,1.8 \mathrm{~Hz}, \mathrm{CH}_{3} \mathrm{CH}=\right), 4.46(2 \mathrm{H}, \mathrm{dt}, J=5.6,1.5 \mathrm{~Hz}$, $\left.-\mathrm{OCH}_{2} \mathrm{CH}=\mathrm{CH}_{2}\right), 5.18\left(2 \mathrm{H}, \mathrm{s}, \mathrm{NCH}_{2} \mathrm{Ph}\right), 5.24\left(1 \mathrm{H}, \mathrm{dq}, J=10.5,1.5 \mathrm{~Hz},-\mathrm{CH}_{2} \mathrm{CH}=\mathrm{CHH}\right), 5.36(1 \mathrm{H}, \mathrm{dq}, J$ $\left.=17.1,1.5 \mathrm{~Hz},-\mathrm{CH}_{2} \mathrm{CH}=\mathrm{CHH}\right), 5.91\left(1 \mathrm{H}, \mathrm{dq}, J=11.2,6.8 \mathrm{~Hz},-\mathrm{CH}=\mathrm{CHCH}_{3}\right), 6.01(1 \mathrm{H}, \mathrm{ddt}, J=17.1$, $\left.10.5,5.6 \mathrm{~Hz},-\mathrm{OCH}_{2} \mathrm{CH}=\mathrm{CH}_{2}\right), 6.01\left(1 \mathrm{H}, \mathrm{br} \mathrm{d}, J=11.2 \mathrm{~Hz}, \mathrm{ArCH}=\mathrm{CHCH}_{3}\right.$, overlapped), $7.07(2 \mathrm{H}, \mathrm{br} \mathrm{d}, J$ $=7.6 \mathrm{~Hz}, \mathrm{Ph}-\mathrm{H}), 7.24(1 \mathrm{H}, \mathrm{br} \mathrm{t}, J=7.6 \mathrm{~Hz}, \mathrm{Ph}-\mathrm{H}), 7.28(1 \mathrm{H}, \mathrm{s}$, pyrazole-H), $7.30(2 \mathrm{H}, \mathrm{br} \mathrm{t}, J=7.6 \mathrm{~Hz}$, Ph-H); ${ }^{13} \mathrm{C}$ NMR $\left(150 \mathrm{MHz}, \mathrm{CDCl}_{3}\right): \delta 15.7,53.9,72.4,115.4,117.4,126.59,126.64,127.5,133.2,137.1$, 142.7 (three signals should be overlapped with signals of the (E)-isomer).

$(E / Z)-4-A l l y l o x y-1-b e n z y l-5-(1-(1-m e t h y l)$ propenyl)-1H-pyrazole (6c): isomer ratio = ca. 1:1; oil; IR (film) $v_{\max } 1562(\mathrm{C}=\mathrm{C}), 1496$ (C=C), $1455(\mathrm{C}=\mathrm{C}) \mathrm{cm}^{-1} ;{ }^{1} \mathrm{H}$ NMR (400 MHz, CDCl $\left.)_{3}\right): \delta 1.43(1.6 \mathrm{H}$, $\left.\mathrm{dd}, J=6.7,1.4 \mathrm{~Hz}, \mathrm{CH}_{3} \mathrm{CH}=\right), 1.72\left(1.4 \mathrm{H}, \mathrm{dd}, J=6.9,1.2 \mathrm{~Hz}, \mathrm{CH}_{3} \mathrm{CH}=\right), 4.414(1.06 \mathrm{H}, \mathrm{d}, J=5.5 \mathrm{~Hz}$, $\left.-\mathrm{OCH}_{2} \mathrm{CH}=\right), 4.417\left(0.94 \mathrm{H}, \mathrm{d}, J=5.5 \mathrm{~Hz},-\mathrm{OCH}_{2} \mathrm{CH}=\right), 5.08\left(0.94 \mathrm{H}, \mathrm{s}, \mathrm{NCH}_{2} \mathrm{Ph}\right), 5.19\left(1.06 \mathrm{H}, \mathrm{s}, \mathrm{NCH}_{2} \mathrm{Ph}\right)$, 5.19-5.36 $\left(2 \mathrm{H}, \mathrm{m},=\mathrm{CH}_{2}\right), 5.54\left(0.44 \mathrm{H}, \mathrm{qq}, J=6.9,1.6 \mathrm{~Hz},-\mathrm{C}\left(\mathrm{CH}_{3}\right) \mathrm{H}=\mathrm{CH}_{3}\right), 5.75(0.56 \mathrm{H}, \mathrm{qq}, J=6.8$, $\left.1.6 \mathrm{~Hz},-\mathrm{C}\left(\mathrm{CH}_{3}\right) \mathrm{H}=\mathrm{CH}_{3}\right), 5.92-6.04\left(1 \mathrm{H}, \mathrm{m},-\mathrm{CH}_{2} \mathrm{CH}=\mathrm{CH}_{2}\right), 7.02(0.94 \mathrm{H}$, br d, J = 7.3 Hz, Ph-H $), 7.07$ $(1.06 \mathrm{H}$, br d, $J=7.2 \mathrm{~Hz}, \mathrm{Ph}-\mathrm{H}), 7.18-7.29(3 \mathrm{H}, \mathrm{m}, \mathrm{Ph}-\mathrm{H}), 7.29\left(1 \mathrm{H}, \mathrm{s}\right.$, pyrazole-H); ${ }^{13} \mathrm{C}$ NMR $(100 \mathrm{MHz}$, $\left.\mathrm{CDCl}_{3}\right): \delta 13.9,15.2,16.0,23.1,53.7,54.0,72.8,117.45,117.54,125.0,126.77,126.86,127.21,127.3,127.5$, 128.46, 128.49, 129.0, 129.2, 129.5, 133.2, 133.7, 133.8, 137.3, 137.8, 141.6, 141.7; HREIMS $m / z$ calcd. for $\mathrm{C}_{17} \mathrm{H}_{20} \mathrm{~N}_{2} \mathrm{O}\left(\mathrm{M}^{+}\right)$268.1576, found 268.1575.

(E/Z)-4-Allyloxy-1-benzyl-5-(1-(1-phenyl)propenyl)-1H-pyrazole (6d): isomer ratio = ca. 7:1; oil; IR (film) $v_{\max } 1556(\mathrm{C}=\mathrm{C}), 1500(\mathrm{C}=\mathrm{C}) \mathrm{cm}^{-1} ;{ }^{1} \mathrm{H}$ NMR $\left(400 \mathrm{MHz}, \mathrm{CDCl}_{3}\right): \delta 1.55(2.7 \mathrm{H}, \mathrm{d}, J=6.9 \mathrm{~Hz}$, $\left.\mathrm{CH}_{3} \mathrm{CH}=\right), 1.85\left(0.3 \mathrm{H}, \mathrm{d}, J=7.3 \mathrm{~Hz}, \mathrm{CH}_{3} \mathrm{CH}=\right), 4.38\left(0.25 \mathrm{H}\right.$, br d, $\left.J=5.5 \mathrm{~Hz},-\mathrm{OCH}_{2} \mathrm{CH}=\right), 4.44$ $\left(1.75 \mathrm{H}\right.$, br d, $\left.J=3.9 \mathrm{~Hz},-\mathrm{OCH}_{2} \mathrm{CH}=\mathrm{CH}_{2}\right), 4.64(1 \mathrm{H}$, br d, $J=14.9 \mathrm{~Hz}, \mathrm{NCHHPh}), 4.94(1 \mathrm{H}$, br d, $J=14.8 \mathrm{~Hz}, \mathrm{NCHHPh}), 5.19\left(1 \mathrm{H}, \mathrm{dd}, J=10.5,1.3 \mathrm{~Hz},-\mathrm{CH}_{2} \mathrm{CH}=\mathrm{CHH}\right), 5.29(1 \mathrm{H}, \mathrm{dq}, J=17.2,1.6 \mathrm{~Hz}$, $\left.-\mathrm{CH}_{2} \mathrm{CH}=\mathrm{CHH}\right), 5.89-6.99\left(1 \mathrm{H}, \mathrm{m},-\mathrm{OCH}_{2} \mathrm{CH}=\mathrm{CH}_{2}\right.$ overlaps with $\left.0.12 \mathrm{H}, \mathrm{m},=\mathrm{CHCH}_{3}\right), 6.31(0.88 \mathrm{H}$, $\left.\mathrm{q}, J=7.0 \mathrm{~Hz},=\mathrm{CHCH}_{3}\right), 6.89-6.90(2 \mathrm{H}, \mathrm{m}, \mathrm{Ph}-\mathrm{H}), 7.08-7.49(8 \mathrm{H}, \mathrm{m}, \mathrm{Ph}-\mathrm{H}), 7.39(1 \mathrm{H}, \mathrm{s}$, pyrazole-H); ${ }^{13} \mathrm{C}$ NMR $\left(100 \mathrm{MHz}, \mathrm{CDCl}_{3}\right): \delta 15.8,54.3,72.7,117.5,126.2,127.0,127.1,127.4,128.27,128.33,128.5$, 129.2, 129.3, 131.2, 133.7, 137.0, 139.7, 143.3; HREIMS $m / z$ calcd. for $\mathrm{C}_{22} \mathrm{H}_{22} \mathrm{~N}_{2} \mathrm{O}\left(\mathrm{M}^{+}\right)$330.1732, found 330.1729 .

Synthesis of (E/Z)-4-allyloxy-1-benzyl-5-(1-(1-methoxycarbonyl)propenyl)-1H-pyrazole (6e) from 2e: To an acetone solution $(4.5 \mathrm{~mL})$ of $2 \mathbf{e}$ with a small amount of $5 \mathbf{e}(121.8 \mathrm{mg}, 0.45 \mathrm{mmol})$ in a microwave vial were added $\mathrm{K}_{2} \mathrm{CO}_{3}(61.8 \mathrm{mg}, 0.45 \mathrm{mmol})$ in water $(0.5 \mathrm{~mL})$ and allyl bromide $(0.04 \mathrm{~mL}$, $0.45 \mathrm{mmol}$ ). After the reaction vial was sealed, the mixture was heated under microwave irradiation at $60{ }^{\circ} \mathrm{C}$ for $1 \mathrm{~h}$. After it had cooled, the reaction was quenched by adding aqueous $\mathrm{NH}_{4} \mathrm{Cl}$. Then, the reaction mixture was extracted with EtOAc three times. The organic layer was washed with brine, dried over $\mathrm{MgSO}_{4}$, filtered, and then evaporated to give a crude residue, which was purified using column chromatography (eluent: hexane:EtOAc $=2: 1)$ to give pure $6 \mathbf{e}(117.1 \mathrm{mg}, 84 \%)$. 
6e (isomer ratio $=$ ca. 13:1): oil; IR (film) $v_{\max } 1717(\mathrm{C}=\mathrm{O}), 1500(\mathrm{C}=\mathrm{C}) \mathrm{cm}^{-1} ;{ }^{1} \mathrm{H}$ NMR: $\delta 1.54$ $\left(2.6 \mathrm{H}, \mathrm{d}, J=7.2 \mathrm{~Hz}, \mathrm{CH}_{3} \mathrm{CH}=\right.$ of major isomer), $2.12\left(0.4 \mathrm{H}, \mathrm{d}, J=7.2 \mathrm{~Hz}, \mathrm{CH}_{3} \mathrm{CH}=\right.$ of minor isomer), $3.52\left(0.4 \mathrm{H}, \mathrm{s},-\mathrm{OCH}_{3}\right.$ of minor isomer), $3.60\left(2.6 \mathrm{H}, \mathrm{s},-\mathrm{OCH}_{3}\right.$ of major isomer $), 4.92(0.93 \mathrm{H}, \mathrm{br} \mathrm{d}$, $J=15.3 \mathrm{~Hz}, \mathrm{NCH} H \mathrm{Ph}$ of major isomer), $5.01\left(0.14 \mathrm{H}, \mathrm{s}, \mathrm{NCH}_{2} \mathrm{Ph}\right.$ of minor isomer $), 5.03(0.93 \mathrm{H}, \mathrm{br} \mathrm{d}$, $J=15.3 \mathrm{~Hz}, \mathrm{NCHHPh}$ of major isomer), $5.11\left(0.93 \mathrm{H}, \mathrm{dq}, J=10.6,1.4 \mathrm{~Hz},-\mathrm{CH}_{2} \mathrm{CH}=\mathrm{CHH}\right.$ of major isomer), $5.14\left(0.07 \mathrm{H}, \mathrm{dq}, J=10.6,1.5 \mathrm{~Hz},-\mathrm{CH}_{2} \mathrm{CH}=\mathrm{CHH}\right.$ of minor isomer $), 5.22(0.93 \mathrm{H}, \mathrm{dq}, J=17.5$, $1.6 \mathrm{~Hz},-\mathrm{CH}_{2} \mathrm{CH}=\mathrm{CHH}$ of major isomer), $5.23\left(1 \mathrm{H}, \mathrm{dq}, J=17.4,1.6 \mathrm{~Hz},-\mathrm{CH}_{2} \mathrm{CH}=\mathrm{CHH}\right.$ of minor isomer), 5.87-5.93 $\left(1 \mathrm{H}, \mathrm{m},-\mathrm{OCH}_{2} \mathrm{CH}=\mathrm{CH}_{2}\right), 6.46\left(0.07 \mathrm{H}, \mathrm{q}, J=7.3 \mathrm{~Hz},-\mathrm{C}_{\mathrm{q}}=\mathrm{CHCH}_{3}\right.$ of minor isomer $), 7.06$ $(2 \mathrm{H}$, br d, $J=6.6 \mathrm{~Hz}, \mathrm{Ph}-\mathrm{H}), 7.17-7.30(3 \mathrm{H}, \mathrm{m}, \mathrm{Ph}-\mathrm{H}), 7.20\left(0.07 \mathrm{H}, \mathrm{q}, J=7.2 \mathrm{~Hz},-\mathrm{C}_{\mathrm{q}}=\mathrm{CHCH}_{3}\right.$ of major isomer), $7.32\left(1 \mathrm{H}, \mathrm{s}\right.$, pyrazole-H); ${ }^{13} \mathrm{C} \mathrm{NMR}\left(100 \mathrm{MHz}, \mathrm{CDCl}_{3}\right): \delta 15.7,52.0,54.6,72.5,117.6,122.0$, 123.2, 126.4, 127.4, 127.6, 128.4, 133.4, 136.7, 143.2, 147.9, 165.9 (minor isomer: 16.2, 51.5, 54.1, 72.8, 121.5, 123.1, 126.6, 127.5, 136.9, 147.7); HREIMS $m / z$ calcd. for $\mathrm{C}_{18} \mathrm{H}_{20} \mathrm{~N}_{2} \mathrm{O}_{3}\left(\mathrm{M}^{+}\right)$312.1474, found 312.1467.

\subsection{Ring-Closing Metathesis of 6 to 1H-1,5-Dihydropyrano[3,2-c]pyrazoles 7 (Table 1)}

General procedure (Table 1, entry 3): To a solution of $\mathbf{6 a}(21.8 \mathrm{mg}, 0.054 \mathrm{mmol})$ in $\mathrm{CH}_{2} \mathrm{Cl}_{2}(2 \mathrm{~mL})$ was added $\mathrm{Grubbs}^{2 \mathrm{nd}}(1.7 \mathrm{mg}, 2.7 \mathrm{mmol})$ at $\mathrm{rt}$. The reaction mixture was stirred at $\mathrm{rt}$ for $1 \mathrm{~h}$, and then the solvent was removed under reduced pressure, affording a crude residue, which was purified using silica gel column chromatography (eluent: EtOAc:hexane = 1:3) to afford 7a (16.2 mg, 83\%).

${ }^{*}$ General procedure for MW-aided reaction (Table 1, entry 5): To a solution of 6a (16.4 mg, $0.04 \mathrm{mmol})$ in $\mathrm{CH}_{2} \mathrm{Cl}_{2}(2 \mathrm{~mL})$ was added $\mathrm{Grubbs}^{2 \mathrm{nd}}(2.3 \mathrm{mg}, 2.0 \mathrm{mmol})$ in a microwave vial. The reaction mixture was heated under microwave irradiation at $80{ }^{\circ} \mathrm{C}$ for $3 \mathrm{~min}$. After the reaction mixture had cooled, the solvent was removed under reduced pressure, affording a crude residue, which was purified using silica gel column chromatography (eluent: EtOAc:hexane $=1: 4$ ) to afford 7a (12.8 mg, 87\%).

1,5-Dihydro-1-tritylpyrano[3,2-c]pyrazole (7a): oil; IR (film) vmax 1677 (C=C), 1581 (C=C), 1493 $(\mathrm{C}=\mathrm{C}), 1447(\mathrm{C}=\mathrm{C}) \mathrm{cm}^{-1},{ }^{1} \mathrm{H}$ NMR $\left(400 \mathrm{MHz} \mathrm{CDCl}_{3}\right): \delta 4.64\left(2 \mathrm{H}, \mathrm{dd}, \mathrm{J}=3.8,1.8 \mathrm{~Hz},-\mathrm{OCH} \mathrm{CH}^{\mathrm{CH}-}\right)$, $5.15\left(1 \mathrm{H}, \mathrm{dt}, J=10.2,3.7 \mathrm{~Hz},-\mathrm{OCH}_{2} \mathrm{CH}=\mathrm{CH}-\right), 5.28\left(1 \mathrm{H}, \mathrm{dtd}, J=10.2,1.8,0.8 \mathrm{~Hz},-\mathrm{OCH}_{2} \mathrm{CH}=\mathrm{CH}-\right)$, 7.08-7.17 (6H, m, Tr-H), $7.18\left(1 \mathrm{H}, \mathrm{d}, J=0.8 \mathrm{~Hz}\right.$, pyrazole-H), 7.23-7.32 (9H, m, Tr-H); ${ }^{13} \mathrm{C}$ NMR (100 $\left.\mathrm{MHz}_{2} \mathrm{CDCl}_{3}\right): \delta 66.9,78.0,117.7,118.6,124.3,127.55,127.57,130.1,141.4,142.7 ;$ HREIMS $m / z$ calcd. for $\mathrm{C}_{25} \mathrm{H}_{20} \mathrm{~N}_{2} \mathrm{O}\left(\mathrm{M}^{+}\right) 364.1575$, found 364.1585.

1-Benzyl-1,5-dihydropyrano[3,2-c]pyrazole (7b): oil; IR (film) $v_{\max } 1566$ (C=C), 1495 (C=C), 1452 $(\mathrm{C}=\mathrm{C}) \mathrm{cm}^{-1} ;{ }^{1} \mathrm{H}$ NMR $\left(400 \mathrm{MHz}, \mathrm{CDCl}_{3}\right): \delta 4.75\left(2 \mathrm{H}, \mathrm{dd}, J=3.9,1.8 \mathrm{~Hz},-\mathrm{OCH}_{2} \mathrm{CH}=\right), 5.21(2 \mathrm{H}, \mathrm{s}$, $\left.\mathrm{ArCH}_{2} \mathrm{Ph}\right), 5.53\left(1 \mathrm{H}, \mathrm{dt}, J=10.0,3.9 \mathrm{~Hz},-\mathrm{OCH}_{2} \mathrm{CH}=\mathrm{CH}-\right), 6.34\left(1 \mathrm{H}, \mathrm{br} \mathrm{d}, J=10.0 \mathrm{~Hz},-\mathrm{OCH}_{2} \mathrm{CH}=\mathrm{CH}-\right)$, $7.10\left(1 \mathrm{H}, \mathrm{d}, J=0.8 \mathrm{~Hz}\right.$, pyrazole-H), 7.10-7.14 (2H, d, J=6.6 Hz, Ph-H), 7.26-7.32 (3H, m, Ph-H); ${ }^{13} \mathrm{C}$ NMR $\left(100 \mathrm{MHz}, \mathrm{CDCl}_{3}\right): \delta$ 54.0, 67.2, 115.5, 119.7, 124.5, 127.1, 127.9, 128.8, 136.6, 140.9; HREIMS m/z calcd. for $\mathrm{C}_{13} \mathrm{H}_{12} \mathrm{~N}_{2} \mathrm{O}\left(\mathrm{M}^{+}\right)$212.0950, found 212.0949 .

1-Benzyl-1,5-dihydro-7-methylpyrano[3,2-c]pyrazole (7c): oil; IR (film) $v_{\max } 1732$ (C=O), 1541 $(\mathrm{C}=\mathrm{C}) \mathrm{cm}^{-1} ;{ }^{1} \mathrm{H}$ NMR $\left(400 \mathrm{MHz}, \mathrm{CDCl}_{3}\right): \delta 1.96\left(3 \mathrm{H}\right.$, br s, $\left.\mathrm{C}_{\mathrm{q}} \mathrm{CH}_{3}\right), 4.64(2 \mathrm{H}, \mathrm{dq}, J=3.3,1.6 \mathrm{~Hz}$, $\left.-\mathrm{OCH}_{2} \mathrm{CH}=\right)$, 5.23-5.26 (1H, m, $\left.-\mathrm{OCH}_{2} \mathrm{CH}=\right), 5.39\left(2 \mathrm{H}, \mathrm{s}, \mathrm{NCH}_{2} \mathrm{Ph}\right), 7.01(2 \mathrm{H}$, br d, J = 7.0 Hz, Ph-H), $7.17\left(1 \mathrm{H}, \mathrm{s}\right.$, pyrazole-H), 7.24-7.32 (3H, m, Ph-H); $\left.{ }^{13} \mathrm{C} \mathrm{NMR} \mathrm{(100} \mathrm{MHz,} \mathrm{CDCl}_{3}\right): \delta 18.1,55.3,67.6$, 116.5, 124.7, 126.0, 127.6, 127.3, 128.7, 137.7, 141.4; HREIMS $m / z$ calcd. for $\mathrm{C}_{15} \mathrm{H}_{14} \mathrm{~N}_{2} \mathrm{O}_{3}\left(\mathrm{M}^{+}\right) 270.1004$, found 270.1003 .

1-Benzyl-1,5-dihydro-7-methoxycarbonylpyrano[3,2-c]pyrazole (7e): oil; IR (film) v $v_{\max } 1732$ (C=O), $1541(\mathrm{C}=\mathrm{C}) \mathrm{cm}^{-1},{ }^{1} \mathrm{H} \mathrm{NMR}\left(400 \mathrm{MHz} \mathrm{CDCl}_{3}\right): \delta 3.76\left(3 \mathrm{H}, \mathrm{s},-\mathrm{COOCH}_{3}\right), 4.72(2 \mathrm{H}, \mathrm{d}, J=4.5 \mathrm{~Hz}$, $\left.-\mathrm{OCH}_{2} \mathrm{CH}=\right), 5.57\left(2 \mathrm{H}, \mathrm{s}, \mathrm{ArCH}_{2} \mathrm{Ph}\right), 6.45\left(1 \mathrm{H}, \mathrm{t}, J=4.5 \mathrm{~Hz},-\mathrm{OCH}_{2} \mathrm{CH}=\mathrm{C}_{\mathrm{q}}\right), 6.33(1 \mathrm{H}, \mathrm{br} \mathrm{d}, J=10.0 \mathrm{~Hz}$, $\left.-\mathrm{OCH}_{2} \mathrm{CH}=\mathrm{CH}-\right), 7.04(2 \mathrm{H}, \mathrm{br} \mathrm{d}, \mathrm{J}=6.5 \mathrm{~Hz}, \mathrm{Ph}-\mathrm{H}), 7.23(1 \mathrm{H}, \mathrm{s}$, pyrazole-H), 7.23-7.31 (3H, m, Ph-H); ${ }^{13} \mathrm{C}$ NMR $\left(100 \mathrm{MHz} \mathrm{CDCl}_{3}\right): \delta 52.3,56.4,66.9,124.0,124.7,127.0,127.4,128.4,128.7,137.5,142.4,163.8$; HREIMS $m / z$ calcd. for $\mathrm{C}_{15} \mathrm{H}_{14} \mathrm{~N}_{2} \mathrm{O}_{3}\left(\mathrm{M}^{+}\right)$270.1004, found 270.1003.

1-Benzyl-1,7-dihydropyrano[3,2-c]pyrazole (8b): oil; IR (film) $v_{\max } 1607$ (C=C), 1586 (C=C), 1557 $(\mathrm{C}=\mathrm{C}) \mathrm{cm}^{-1} ;{ }^{1} \mathrm{H} \mathrm{NMR}\left(400 \mathrm{MHz}_{\mathrm{CDCl}}\right): \delta 3.23\left(2 \mathrm{H}, \mathrm{dd}, J=3.3,2.0 \mathrm{~Hz}, \mathrm{ArCH} \mathrm{CH}^{\mathrm{C}}\right), 4.77(1 \mathrm{H}$, 
$\left.\mathrm{dt}, J=6.3,3.4 \mathrm{~Hz},-\mathrm{CH}_{2} \mathrm{CH}=\mathrm{CH}-\right), 5.17\left(2 \mathrm{H}, \mathrm{s}, \mathrm{ArCH}_{2} \mathrm{Ph}\right), 6.42(1 \mathrm{H}, \mathrm{dt}, J=6.2,2.0 \mathrm{~Hz},=\mathrm{CH}=\mathrm{CHO}-)$, 7.06-7.20 (2H, m, Ph-H), 7.22-7.33 (4H, m, Ph-H, pyrazole-H); ${ }^{13} \mathrm{C}$ NMR (100 MHz, $\left.\mathrm{CDCl}_{3}\right): \delta 19.5$, 53.8, 97.1, 125.1, 126.4 127.0, 127.9, 128.8, 129.0, 136.6, 141.3; HREIMS $m / z$ calcd. for $\mathrm{C}_{13} \mathrm{H}_{12} \mathrm{~N}_{2} \mathrm{O}\left(\mathrm{M}^{+}\right)$ 212.0950, found 212.0947 .

1-Benzyl-1,7-dihydro-7-methylenepyrano[3,2-c]pyrazole (9c): oil; IR (film) $v_{\max } 1644$ (C=C), 1556 $(\mathrm{C}=\mathrm{C}), 1401(\mathrm{C}=\mathrm{C}) \mathrm{cm}^{-1} ;{ }^{1} \mathrm{H}$ NMR $\left(400 \mathrm{MHz}, \mathrm{CDCl}_{3}\right): \delta 2.54\left(2 \mathrm{H}, \mathrm{br} \mathrm{t}, J=5.6 \mathrm{~Hz},-\mathrm{OCH}_{2} \mathrm{CH}_{2} \mathrm{C}_{\mathrm{q}}\right), 4.17$ $\left(2 \mathrm{H}, \mathrm{t}, J=5.7 \mathrm{~Hz},-\mathrm{OCH}_{2} \mathrm{CH}_{2}-\right), 4.78\left(1 \mathrm{H}, \mathrm{br} \mathrm{s}, \mathrm{C}_{\mathrm{q}} \mathrm{CHH}\right), 4.96\left(1 \mathrm{H}, \mathrm{br} \mathrm{s}, \mathrm{C}_{\mathrm{q}} \mathrm{CHH}\right), 5.43\left(2 \mathrm{H}, \mathrm{s}, \mathrm{NCH}_{2} \mathrm{Ph}\right)$, $7.02(2 \mathrm{H}, \mathrm{d}, J=7.0 \mathrm{~Hz}, \mathrm{Ph}-\mathrm{H}), 7.22-7.32\left(4 \mathrm{H}, \mathrm{m}, \mathrm{Ph}-\mathrm{H}\right.$, pyrazole-H); ${ }^{13} \mathrm{C}$ NMR $\left(100 \mathrm{MHz}, \mathrm{CDCl}_{3}\right): \delta 32.2$, $55.4,68.3,107.2,124.0,125.3,126.2,127.5,128.7,129.7,136.9,142.6$; HREIMS $m / z$ calcd. for $\mathrm{C}_{14} \mathrm{H}_{14} \mathrm{~N}_{2} \mathrm{O}$ $\left(\mathrm{M}^{+}\right)$226.1106, found 226.1102.

1,4-Bis((1-benzyl-5-(1-phenylprop-1-en-1-yl)-1H-pyrazol-4-yl)oxy)but-2-ene (10d): oil; IR (film) $v_{\max } 1569(\mathrm{C}=\mathrm{C}), 1496(\mathrm{C}=\mathrm{C}) \mathrm{cm}^{-1} ;{ }^{1} \mathrm{H}$ NMR $\left(400 \mathrm{MHz} \mathrm{CDCl}_{3}\right): \delta 1.51\left(6 \mathrm{H}, \mathrm{d}, J=7.1 \mathrm{~Hz},=\mathrm{CHCH}_{3}\right)$, $4.40\left(4 \mathrm{H}, \mathrm{br} \mathrm{s},-\mathrm{OCH}_{2} \mathrm{CH}=\right), 4.62(2 \mathrm{H}, \mathrm{br} \mathrm{d}, J=14.8 \mathrm{~Hz}, \operatorname{ArCHHPh}), 4.92(2 \mathrm{H}, \mathrm{br} \mathrm{d}, J=14.4 \mathrm{~Hz}$, $\operatorname{ArCHHPh}), 5.82-5.84\left(2 \mathrm{H}, \mathrm{m},-\mathrm{OCH}_{2} \mathrm{CH}=\right), 6.29\left(2 \mathrm{H}, \mathrm{q}, J=7.1 \mathrm{~Hz},=\mathrm{CHCH}_{3}\right), 6.92-6.95(4 \mathrm{H}, \mathrm{m}, \mathrm{Ph}-\mathrm{H})$, 7.06-7.25 (6H, m, Ph-H), $7.35(2 \mathrm{H}, \mathrm{s}$, pyrazole-H) $){ }^{13} \mathrm{C}$ NMR $\left(100 \mathrm{MHz}, \mathrm{CDCl}_{3}\right): \delta 15.8,54.3,71.6$, $126.1,127.1,127.35,127.42,128.3,128.7,129.1,129.2,131.2,137.0,140.0,143.2$ (three carbon signals overlapped); HREIMS $m / z$ calcd. for $\mathrm{C}_{42} \mathrm{H}_{40} \mathrm{~N}_{4} \mathrm{O}_{2}\left(\mathrm{M}^{+}\right)$632.3151, found 632.3145.

1,4-Bis((1-benzyl-5-(1-(methoxycarbonyl)prop-1-en-1-yl)-1H-pyrazol-4-yl)oxy)but-2-ene (10e): oil; IR (film) $v_{\max } 1722(\mathrm{C}=\mathrm{O}), 1712(\mathrm{C}=\mathrm{O}), 1642(\mathrm{C}=\mathrm{C}), 1573(\mathrm{C}=\mathrm{C}) \mathrm{cm}^{-1},{ }^{1} \mathrm{H}$ NMR $\left(400 \mathrm{MHz}, \mathrm{CDCl}_{3}\right)$ : $\delta 1.54\left(5.4 \mathrm{H}, \mathrm{d}, J=7.0 \mathrm{~Hz},=\mathrm{CHCH}_{3}\right.$ of major isomer $), 2.14\left(0.6 \mathrm{H}, \mathrm{d}, J=7.2 \mathrm{~Hz},=\mathrm{CHCH}_{3}\right.$ of minor isomer), $3.61\left(0.6 \mathrm{H}, \mathrm{s},-\mathrm{OCH}_{3}\right.$ of minor isomer), $3.62\left(5.4 \mathrm{H}, \mathrm{s},=\mathrm{CHCH}_{3}\right.$ of major isomer $), 4.42(3.6 \mathrm{H}$, br s, $-\mathrm{OCH}_{2} \mathrm{CH}=$ of major isomer), $4.48\left(0.4 \mathrm{H}, \mathrm{br} \mathrm{s},-\mathrm{OCH}_{2} \mathrm{CH}=\right.$ of minor isomer $), 5.08(1.8 \mathrm{H}, \mathrm{br} \mathrm{d}$, $J=13.3 \mathrm{~Hz}, \mathrm{ArCH} H \mathrm{Ph}$ of major isomer $), 5.11\left(0.4 \mathrm{H}, \mathrm{s}, \mathrm{ArCH}_{2} \mathrm{Ph}\right.$ of minor isomer $), 5.12(1.8 \mathrm{H}, \mathrm{br} \mathrm{d}$, $J=13.3 \mathrm{~Hz}$, ArCHHPh of major isomer), $5.77\left(3.6 \mathrm{H}, \mathrm{br} \mathrm{t}, J=3.7 \mathrm{~Hz},-\mathrm{OCH}_{2} \mathrm{CH}=\right.$ of minor isomer), $5.88\left(0.4 \mathrm{H}, \mathrm{br} \mathrm{t}, J=3.7 \mathrm{~Hz},-\mathrm{OCH}_{2} \mathrm{CH}=\right.$ of major isomer $), 6.28\left(0.2 \mathrm{H}, \mathrm{q}, J=7.5 \mathrm{~Hz},=\mathrm{CHCH}_{3}\right.$ of minor isomer), $7.08(4 \mathrm{H}, \mathrm{d}, J=6.8 \mathrm{~Hz}, \mathrm{Ph}-\mathrm{H}), 7.20-7.32\left(7.8 \mathrm{H}, \mathrm{m}, \mathrm{Ph}-\mathrm{H},=\mathrm{CHCH}_{3}\right.$ of major isomer $), 7.33(2 \mathrm{H}, \mathrm{s}$, pyrazole-H); ${ }^{13} \mathrm{C}$ NMR $\left(100 \mathrm{MHz}, \mathrm{CDCl}_{3}\right): \delta 15.7,52.1,54.6,71.5,122.1,123.2,126.4,127.4,127.6,128.4$, 128.5, 136.7, 147.9, 165.9; HREIMS $m / z$ calcd. for $\mathrm{C}_{34} \mathrm{H}_{36} \mathrm{~N}_{4} \mathrm{O}_{6}\left(\mathrm{M}^{+}\right)$596.2635, found 596.2634.

Methyl 2-(1-benzyl-4-(cinnamyloxy)-1H-pyrazol-5-yl)but-2-enoate (11e): oil; IR (film) $v_{\max } 1716$ $(\mathrm{C}=\mathrm{O}), 1644(\mathrm{C}=\mathrm{C}), 1574(\mathrm{C}=\mathrm{C}) \mathrm{cm}^{-1} ;{ }^{1} \mathrm{H} \mathrm{NMR}\left(600 \mathrm{MHz}, \mathrm{CDCl}_{3}\right): \delta 1.58\left(3 \mathrm{H}, \mathrm{d}, J=7.3 \mathrm{~Hz},=\mathrm{CHCH}_{3}\right)$, $3.58\left(3 \mathrm{H}, \mathrm{s},-\mathrm{COOCH}_{3}\right), 4.58\left(2 \mathrm{H}, \mathrm{d}, J=6.2 \mathrm{~Hz},-\mathrm{OCH}_{2} \mathrm{CH}=\right), 5.02(1 \mathrm{H}, \mathrm{br} \mathrm{d}, J=15.2 \mathrm{~Hz}, \mathrm{ArCHHPh})$, $5.12(1 \mathrm{H}, \mathrm{brd}, J=15.2 \mathrm{~Hz}, \mathrm{ArCHHPh}), 6.30\left(1 \mathrm{H}, \mathrm{dt}, J=15.9,6.2 \mathrm{~Hz},-\mathrm{OCH}_{2} \mathrm{CH}=\mathrm{CH}-\right), 6.62(1 \mathrm{H}, \mathrm{d}, J=$ $15.9 \mathrm{~Hz},-\mathrm{CH}=\mathrm{CHPh}), 7.09(2 \mathrm{H}, \mathrm{d}, J=7.3 \mathrm{~Hz}, \mathrm{Ph}-\mathrm{H}), 7.20-7.38(8 \mathrm{H}, \mathrm{m}, \mathrm{Ph}-\mathrm{H}), 7.30(1 \mathrm{H}, \mathrm{q}, J=7.3 \mathrm{~Hz}$, $\left.-\mathrm{C}_{\mathrm{q}}=\mathrm{CHCH}_{3}\right), 7.40\left(1 \mathrm{H}\right.$, s, pyrazole-H); ${ }^{13} \mathrm{C} \mathrm{NMR}\left(150 \mathrm{MHz}, \mathrm{CDCl}_{3}\right): \delta 15.8,52.0,54.7,72.7,122.2,123.6$, 124.7, 126.6, 126.9, 127.4, 127.6, 127.9, 128.4, 128.6, 133.1, 136.4, 136.7, 143.2, 147.9, 165.9; HREIMS $\mathrm{m} / \mathrm{z}$ calcd. for $\mathrm{C}_{24} \mathrm{H}_{24} \mathrm{~N}_{2} \mathrm{O}_{3}\left(\mathrm{M}^{+}\right) 388.1787$, found 388.1785.

\subsection{Synthesis of 5-Allyl-4-(2-haloethoxy)-1H-pyrazoles (12) (Scheme 2)}

General procedure: To a solution of $2 \mathbf{a}(50.8 \mathrm{mg}, 0.14 \mathrm{mmol})$ in acetone $(2 \mathrm{~mL})$ in a microwave vial were added 1,2-dibromoethane $(0.05 \mathrm{~mL}, 0.56 \mathrm{mmol}), 20 \%$ aqueous $\mathrm{NaOH}(0.11 \mathrm{~mL}, 0.56 \mathrm{mmol})$, and a catalytic amount of tetrabutylammonium bromide. The sealed reaction vial was MW irradiated at $140{ }^{\circ} \mathrm{C}$ for $30 \mathrm{~min}$. After it had cooled, the reaction mixture was quenched with saturated aqueous $\mathrm{NH}_{4} \mathrm{Cl}$ and extracted with $\mathrm{CH}_{2} \mathrm{Cl}_{2}$. The separated organic layer was dried over $\mathrm{MgSO}_{4}$, filtered, and evaporated under reduced pressure to afford a crude residue. The residue was purified using silica gel column chromatography (eluent: EtOAc:hexane $=1: 3)$ to afford 12a $(42.9 \mathrm{mg}, 65 \%)$ as an oil.

5-Allyl-4-(2-bromoethoxy)-1H-1-tritylpyrazole (12a): pale yellow crystals $\left(\mathrm{CH}_{2} \mathrm{Cl}_{2}\right) ; \mathrm{mp}$ 135-140 ${ }^{\circ} \mathrm{C}$; IR (film) $v_{\max } 1581(\mathrm{C}=\mathrm{C}), 1491(\mathrm{C}=\mathrm{C}), 1446(\mathrm{C}=\mathrm{C}) \mathrm{cm}^{-1} ;{ }^{1} \mathrm{H}$ NMR $\left(500 \mathrm{MHz}, \mathrm{CDCl}_{3}\right)$ : $\delta 2.85\left(2 \mathrm{H}, \mathrm{dt}, J=6.5,1.2 \mathrm{~Hz}, \mathrm{ArCH}_{2} \mathrm{CH}=\mathrm{CH}_{2}\right), 3.56\left(2 \mathrm{H}, \mathrm{t}, J=6.2 \mathrm{~Hz},-\mathrm{OCH}_{2} \mathrm{CH}_{2} \mathrm{CBr}\right), 4.20(2 \mathrm{H}, \mathrm{t}$, $\left.J=6.2 \mathrm{~Hz},-\mathrm{OCH}_{2} \mathrm{CH}_{2} \mathrm{Br}\right), 4.63(1 \mathrm{H}, \mathrm{dq}, J=17.0,1.6 \mathrm{~Hz},-\mathrm{CH}=\mathrm{CHH}), 4.66(1 \mathrm{H}, \mathrm{dq}, J=10.0,1.4 \mathrm{~Hz}$, $-\mathrm{CH}=\mathrm{CHH}), 4.97\left(1 \mathrm{H}, \mathrm{ddt}, J=17.0,10.0,6.5 \mathrm{~Hz},-\mathrm{CH}_{2} \mathrm{CH}=\mathrm{CH}_{2}\right), 7.10-7.13(6 \mathrm{H}, \mathrm{m}, \mathrm{Tr}-\mathrm{H}), 7.25-7.30$ 
(9H, m, Tr-H), 7.33 (1H, s, pyrazole-H); ${ }^{13} \mathrm{C}$ NMR (125 MHz, $\left.\mathrm{CDCl}_{3}\right): \delta$ 29.4, 31.2, 71.6, 78.7, 115.9, 125.6, 127.4, 127.6, 129.9, 130.1, 132.4, 142.8, 143.6; HREIMS $m / z$ calcd. for $\mathrm{C}_{27} \mathrm{H}_{25} \mathrm{BrN}_{2} \mathrm{O}\left(\mathrm{M}^{+}\right)$472.1151, found 472.1149 .

5-Allyl-1-benzyl-4-(2-bromoethoxy)-1H-pyrazole (12b): oil; IR (film) $v_{\max } 1583$ (C=C), 1496 (C=C) $\mathrm{cm}^{-1} ;{ }^{1} \mathrm{H}$ NMR $\left(400 \mathrm{MHz}, \mathrm{CDCl}_{3}\right): \delta 3.29\left(2 \mathrm{H}, \mathrm{dd}, J=4.7,1.7 \mathrm{~Hz}, \mathrm{ArCH}_{2} \mathrm{CH}=\right), 3.56(2 \mathrm{H}, \mathrm{br} \mathrm{t}, J=6.2 \mathrm{~Hz}$, $\left.-\mathrm{OCH}_{2} \mathrm{CH}_{2} \mathrm{Br}\right), 4.20\left(2 \mathrm{H}, \mathrm{brt}, J=6.2 \mathrm{~Hz},-\mathrm{OCH}_{2} \mathrm{CH}_{2} \mathrm{Br}\right), 5.00(1 \mathrm{H}, \mathrm{dd}, J=7.0,1.4 \mathrm{~Hz},-\mathrm{CH}=\mathrm{CHH}), 5.07$ $(1 \mathrm{H}, \mathrm{dd}, J=10.2,1.4 \mathrm{~Hz},-\mathrm{CH}=\mathrm{CH}), 5.73-5.83\left(1 \mathrm{H}, \mathrm{m},-\mathrm{CH}_{2} \mathrm{CH}=\mathrm{CH}_{2}\right), 7.06(2 \mathrm{H}, \mathrm{br} \mathrm{d}, J=8.1 \mathrm{~Hz}$, Bn-H), 7.25-7.33 (4H, m, Ph-H, pyrazole-H); ${ }^{13} \mathrm{C}$ NMR (100 MHz, $\left.\mathrm{CDCl}_{3}\right): \delta 27.1,29.5,53.9,72.4,116.5$, 126.7, 127.2, 127.7, 127.8, 128.7, 133.6, 136.9, 141.6; HREIMS $m / z$ calcd. for $\mathrm{C}_{15} \mathrm{H}_{17} \mathrm{BrN}_{2} \mathrm{O}\left(\mathrm{M}^{+}\right)$320.0524, found 320.0520 .

5-Allyl-4-(2-chloroethoxy)-1H-1-tritylpyrazole $\left(\mathbf{1 2}_{\mathrm{Cl}}\right)$ : white powder $\left(\mathrm{CH}_{2} \mathrm{Cl}_{2}\right) ; \mathrm{mp} 120-125{ }^{\circ} \mathrm{C}$; IR $(\mathrm{KBr}) v_{\max } 1580(\mathrm{C}=\mathrm{C}), 1493(\mathrm{C}=\mathrm{C}), 1446(\mathrm{C}=\mathrm{C}) \mathrm{cm}^{-1}$; ${ }^{1} \mathrm{H}$ NMR $\left(500 \mathrm{MHz}, \mathrm{CDCl}_{3}\right): \delta 2.85(2 \mathrm{H}, \mathrm{br} \mathrm{d}, J=$ $\left.7.6 \mathrm{~Hz}, \mathrm{ArCH}_{2} \mathrm{CH}=\right), 3.72\left(2 \mathrm{H}, \mathrm{t}, J=5.7 \mathrm{~Hz},-\mathrm{OCH}_{2} \mathrm{CH}_{2} \mathrm{Cl}\right), 4.14\left(2 \mathrm{H}, \mathrm{t}, J=5.7 \mathrm{~Hz},-\mathrm{OCH}_{2} \mathrm{CH}_{2} \mathrm{CCl}\right), 4.63$ $(1 \mathrm{H}, \mathrm{dq}, J=17.0,1.6 \mathrm{~Hz},-\mathrm{CH}=\mathrm{CH} H), 4.66(1 \mathrm{H}, \mathrm{dq}, J=10.0,1.4 \mathrm{~Hz},-\mathrm{CH}=\mathrm{CH} H), 4.97(1 \mathrm{H}, \mathrm{ddt}, J=17.0$, 10.0, $\left.6.7 \mathrm{~Hz},-\mathrm{CH}_{2} \mathrm{CH}=\mathrm{CH}_{2}\right), 7.10-7.14(6 \mathrm{H}, \mathrm{m}, \mathrm{Tr}-\mathrm{H}), 7.24-7.31(9 \mathrm{H}, \mathrm{m}, \mathrm{Tr}-\mathrm{H}), 7.34(1 \mathrm{H}, \mathrm{s}$, pyrazole-H); ${ }^{13} \mathrm{C}$ NMR $\left(125 \mathrm{MHz}, \mathrm{CDCl}_{3}\right): \delta 31.2,42.1,71.7,78.6,115.8,125.5,127.3,127.6,129.9,130.0,132.4,142.8$, 143.7; HREIMS $m / z$ calcd. for $\mathrm{C}_{27} \mathrm{H}_{25} \mathrm{ClN}_{2} \mathrm{O}\left(\mathrm{M}^{+}\right) 428.1655$, found 428.1654 . * $\mathrm{MW}$ conditions: $160{ }^{\circ} \mathrm{C}$, $30 \mathrm{~min}$.

\subsection{Reaction of $\mathbf{1 2}$ with Potassium Tert-Butoxide (Table 2, Scheme 2)}

General procedure (Table 2, entry 7): To a solution of 12a $(28.8 \mathrm{mg}, 0.05 \mathrm{mmol})$ in anhydrous THF:t-BuOH $(2 \mathrm{~mL}: 0.5 \mathrm{~mL})$ in a microwave vial was added potassium tert-butoxide $(28.8 \mathrm{mg}$, $0.26 \mathrm{mmol})$. The sealed reaction vial was MW irradiated at $130{ }^{\circ} \mathrm{C}$ for $1 \mathrm{~h}$. After it had cooled, the reaction mixture was quenched with saturated aqueous $\mathrm{NH}_{4} \mathrm{Cl}$ and extracted with $\mathrm{CH}_{2} \mathrm{Cl}_{2}$. The separated organic layer was dried over $\mathrm{MgSO}_{4}$, filtered, and evaporated under reduced pressure to afford a crude residue. The residue was purified using silica gel column chromatography (eluent: EtOAc:hexane $=1: 3)$ to afford 13a $(20.8 \mathrm{mg}, 87 \%)$.

5-Allyl-1-trityl-1H-4-vinyloxypyrazole (13a): white powder $\left(\mathrm{CH}_{2} \mathrm{Cl}_{2}\right) ; \mathrm{mp} 75-80{ }^{\circ} \mathrm{C}$; $\mathrm{IR}(\mathrm{KBr})$ $v_{\max } 1639(\mathrm{C}=\mathrm{C}), 1624(\mathrm{C}=\mathrm{C}), 1566(\mathrm{C}=\mathrm{C}) \mathrm{cm}^{-1},{ }^{1} \mathrm{H}$ NMR $\left(600 \mathrm{MHz}, \mathrm{CDCl}_{3}\right): \delta 2.81(2 \mathrm{H}, \mathrm{ddd}, J=$ $\left.6.8,1.5,1.2 \mathrm{~Hz}, \mathrm{ArCH}_{2} \mathrm{CH}=\mathrm{CH}_{2}\right), 4.23(1 \mathrm{H}, \mathrm{dd}, J=5.4,1.8 \mathrm{~Hz},-\mathrm{OCH}=\mathrm{CHH}), 4.50(1 \mathrm{H}, \mathrm{dd}, J=13.8$, $\left.2.1 \mathrm{~Hz},-\mathrm{OCH}_{2}=\mathrm{CHH}\right), 4.62\left(1 \mathrm{H}, \mathrm{dq}, J=16.7,1.5 \mathrm{~Hz},-\mathrm{CH}_{2} \mathrm{CH}=\mathrm{CHH}\right), 4.68(1 \mathrm{H}, \mathrm{dq}, J=10.9,1.5 \mathrm{~Hz}$, $\left.-\mathrm{CH}_{2} \mathrm{CH}=\mathrm{CHH}\right), 4.99\left(1 \mathrm{H}, \mathrm{ddt}, J=16.5,10.9,2.1 \mathrm{~Hz},-\mathrm{CH}_{2} \mathrm{CH}=\mathrm{CH}_{2}\right), 6.53(1 \mathrm{H}, \mathrm{dd}, J=13.8,6.5 \mathrm{~Hz}$, $\left.-\mathrm{OCH}=\mathrm{CH}_{2}\right), 7.12-7.14(6 \mathrm{H}, \mathrm{m}, \mathrm{Tr}-\mathrm{H}), 7.25-7.31(9 \mathrm{H}, \mathrm{m}, \mathrm{Tr}-\mathrm{H}), 7.40\left(1 \mathrm{H}, \mathrm{s}\right.$, pyrazole-H); ${ }^{13} \mathrm{C}$ NMR $(150$ $\left.\mathrm{MHz}, \mathrm{CDCl}_{3}\right): \delta 31.1,77.8,91.3,116.1,127.4,127.6,128.3,130.0,131.7,131.9,140.4,142.3,150.7$; HREIMS $m / z$ calcd. for $\mathrm{C}_{27} \mathrm{H}_{24} \mathrm{~N}_{2} \mathrm{O}\left(\mathrm{M}^{+}\right)$392.1888, found 392.1880.

5-(1-Propenyl)-1-trityl-1 $\mathrm{H}$-4-vinyloxypyrazole (14a): white powder $\left(\mathrm{CH}_{2} \mathrm{Cl}_{2}\right) ; \mathrm{mp} 133-135{ }^{\circ} \mathrm{C}$; IR (KBr) $v_{\max } 1639(\mathrm{C}=\mathrm{C}), 1560(\mathrm{C}=\mathrm{C}), 1492(\mathrm{C}=\mathrm{C}) \mathrm{cm}^{-1}{ }^{1}{ }^{1} \mathrm{H}$ NMR $\left(600 \mathrm{MHz}, \mathrm{CDCl}_{3}\right): \delta 1.39(3 \mathrm{H}$, $\left.\mathrm{dd}, J=6.8,1.8 \mathrm{~Hz},-\mathrm{CH}=\mathrm{CHCH}_{3}\right), 4.29(1 \mathrm{H}, \mathrm{dd}, J=6.2,2.0 \mathrm{~Hz},-\mathrm{OCH}=\mathrm{CHH}), 4.59(1 \mathrm{H}, \mathrm{dd}, J=13.8$, $2.0 \mathrm{~Hz},-\mathrm{OCH}=\mathrm{CHH}), 5.39\left(1 \mathrm{H}, \mathrm{br} \mathrm{dq}, J=15.8,0.8 \mathrm{~Hz},-\mathrm{CH}=\mathrm{CHCH}_{3}\right), 5.98(1 \mathrm{H}, \mathrm{dq}, J=15.8,6.8 \mathrm{~Hz}$, $\left.-\mathrm{CH}=\mathrm{CHCH}_{3}\right), 6.56\left(1 \mathrm{H}, \mathrm{dd}, J=13.8,6.2 \mathrm{~Hz},-\mathrm{OCH}=\mathrm{CH}_{2}\right), 7.11-7.15(6 \mathrm{H}, \mathrm{m}, \mathrm{Tr}-\mathrm{H}), 7.26-7.32(9 \mathrm{H}$, m, Tr-H), 7.38 (1H, br s, pyrazole-H); ${ }^{13} \mathrm{C}$ NMR (150 MHz, $\left.\mathrm{CDCl}_{3}\right): \delta 18.8,79.8,92.3,119.1,127.38$, 127.44, 128.0, 129.1, 130.3, 131.2, 139.5, 142.7, 150.4; HREIMS $m / z$ calcd. for $\mathrm{C}_{27} \mathrm{H}_{24} \mathrm{~N}_{2} \mathrm{O}\left(\mathrm{M}^{+}\right)$392.1889, found 392.1887 .

(E/Z)-1-Benzyl-5-(1-propenyl)-1H-4-vinyloxypyrazole (14b): $E / Z$ ratio = ca. 5:1; oil; IR (film) $v_{\max } 1642(\mathrm{C}=\mathrm{C}), 1562(\mathrm{C}=\mathrm{C}), 1493(\mathrm{C}=\mathrm{C}) \mathrm{cm}^{-1} ;{ }^{1} \mathrm{H}$ NMR $\left(400 \mathrm{MHz}, \mathrm{CDCl}_{3}\right): \delta 1.68(0.5 \mathrm{H}, \mathrm{d}, J=6.3 \mathrm{~Hz}$, $=\mathrm{CHCH}_{3}$ of $(\mathrm{Z})$-isomer $), 1.82\left(2.5 \mathrm{H}, \mathrm{dd}, J=6.6,1.6 \mathrm{~Hz},=\mathrm{CHCH}_{3}\right.$ of $(E)$-isomer $), 4.22(0.17 \mathrm{H}, \mathrm{dd}, J=$ $6.3,2.0 \mathrm{~Hz},-\mathrm{CH}=\mathrm{CHH}$ of $(\mathrm{Z})$-isomer $), 4.29(0.83 \mathrm{H}, \mathrm{dd}, J=6.3,2.0 \mathrm{~Hz},-\mathrm{CH}=\mathrm{CHH}$ of $(E)$-isomer $), 4.57$ $(0.17 \mathrm{H}, \mathrm{dd}, J=13.7,2.0 \mathrm{~Hz},-\mathrm{CH}=\mathrm{CH} H$ of $(\mathrm{Z})$-isomer $), 4.59(0.83 \mathrm{H}, \mathrm{dd}, J=13.7,2.0 \mathrm{~Hz},-\mathrm{CH}=\mathrm{CH} H$ of (E)-isomer), $5.93\left(0.17 \mathrm{H}, \mathrm{dq}, J=11.0,6.5 \mathrm{~Hz},-\mathrm{CH}=\mathrm{CHCH}_{3}\right.$ of $(\mathrm{Z})$-isomer $), 5.98(0.17 \mathrm{H}, \mathrm{br} \mathrm{d}, J=11.0 \mathrm{~Hz}$, $\mathrm{ArCH}=\mathrm{CHCH}_{3}$ of $(\mathrm{Z})$-isomer), $6.11\left(0.83 \mathrm{H}, \mathrm{br} \mathrm{dq}, J=16.0,1.6 \mathrm{~Hz}, \mathrm{ArCH}=\mathrm{CHCH}_{3}\right.$ of $(E)$-isomer), 6.34 
$\left(0.83 \mathrm{H}, \mathrm{dq}, J=15.8,6.8 \mathrm{~Hz},-\mathrm{CH}=\mathrm{CHCH}_{3}\right.$ of $(E)$-isomer $), 6.49\left(0.17 \mathrm{H}, \mathrm{dd}, J=13.7,6.3 \mathrm{~Hz},-\mathrm{OCH}=\mathrm{CH}_{2}\right.$ of (Z)-isomer), $6.55\left(0.83 \mathrm{H}, \mathrm{dd}, J=13.7,6.3 \mathrm{~Hz},-\mathrm{OCH}=\mathrm{CH}_{2}\right.$ of $(E)$-isomer), $7.07(2 \mathrm{H}, \mathrm{br} \mathrm{d}, J=7.0 \mathrm{~Hz}, \mathrm{Ph}-\mathrm{H})$, 7.23-7.37 (3H, m, Ph-H), 7.33 (1H, br s, pyrazole-H); $\left.{ }^{13} \mathrm{C} \mathrm{NMR} \mathrm{(150} \mathrm{MHz,} \mathrm{CDCl}_{3}\right): \delta 16.0$ (minor), 19.3, 53.4 (minor), 54.0, 91.9 (minor), 92.3, 114.7 (minor), 115.9, 126.5, 126.9, 127.7, 128.7, 128.8, 129.0 (minor), 131.5, 134.2 (minor), 136.9, 138.5 (minor), 150.4 (minor), 150.5; HREIMS $m / z$ calcd. for $\mathrm{C}_{15} \mathrm{H}_{16} \mathrm{~N}_{2} \mathrm{O}$ $\left(\mathrm{M}^{+}\right)$240.1263, found 240.1256 .

(E/Z)-4-(2-Methoxy)ethoxy-3-(1-propenyl)-2H-2-tritylpyrazole (15): oil; IR (film) $v_{\max } 1492$ (C=C), $1446(\mathrm{C}=\mathrm{C}) \mathrm{cm}^{-1} ;{ }^{1} \mathrm{H}$ NMR $\left(400 \mathrm{MHz}, \mathrm{CDCl}_{3}\right): \delta 1.39\left(3 \mathrm{H}, \mathrm{d}, J=6.7 \mathrm{~Hz},=\mathrm{CHCH}_{3}\right), 3.65(0.5 \mathrm{H}, \mathrm{br} \mathrm{t}, J=$ $\left.4.1 \mathrm{~Hz},-\mathrm{OCH}_{2} \mathrm{CH}_{2} \mathrm{Br}\right), 3.7\left(1.5 \mathrm{H}, \mathrm{br} \mathrm{t}, J=4.1 \mathrm{~Hz},-\mathrm{CH}_{2} \mathrm{CH}_{2} \mathrm{Br}\right), 4.07\left(0.5 \mathrm{H}, \mathrm{br} \mathrm{t}, J=3.9 \mathrm{~Hz},-\mathrm{CH}_{2} \mathrm{CH}_{2} \mathrm{Br}\right)$, $3.70\left(1.5 \mathrm{H}, \mathrm{brt}, J=4.1 \mathrm{~Hz},-\mathrm{OCH}_{2} \mathrm{CH}_{2} \mathrm{Br}\right), 5.44(1 \mathrm{H}, \mathrm{br} \mathrm{d}, J=15.8 \mathrm{~Hz},(E)-\mathrm{ArCH}=\mathrm{CH}-), 6.09-6.18(1 \mathrm{H}$, $\left.\mathrm{m},-\mathrm{CH}=\mathrm{CHCH}_{3}\right), 7.11-7.20(6 \mathrm{H}, \mathrm{m}, \mathrm{Tr}-\mathrm{H}), 7.24-7.29(9 \mathrm{H}, \mathrm{m}, \mathrm{Tr}-\mathrm{H}), 7.32\left(1 \mathrm{H}, \mathrm{s}\right.$, pyrazole-H); ${ }^{13} \mathrm{C}$ NMR $\left(150 \mathrm{MHz}, \mathrm{CDCl}_{3}\right): \delta 18.9,59.2,70.6,71.3,79.0,119.9,124.8,127.3,127.4,127.6,130.1,130.4,142.9,143.7$; HREIMS $m / z$ calcd. for $\mathrm{C}_{28} \mathrm{H}_{28} \mathrm{~N}_{2} \mathrm{O}_{2}\left(\mathrm{M}^{+}\right) 424.2151$, found 424.2157 .

4-(2-Methoxy)ethoxy-3-(2-propenyl)-2H-2-tritylpyrazole (16): oil; IR (film) $v_{\max } 1580$ (C=C), 1447 $(\mathrm{C}=\mathrm{C}) \mathrm{cm}^{-1} ;{ }^{1} \mathrm{H} \mathrm{NMR}\left(600 \mathrm{MHz}, \mathrm{CDCl}_{3}\right): \delta 2.84\left(2 \mathrm{H}, \mathrm{br} \mathrm{d}, J=6.5 \mathrm{~Hz}, \mathrm{ArCH}_{2} \mathrm{CH}=\right), 3.41\left(3 \mathrm{H}, \mathrm{s},-\mathrm{OCH}_{3}\right)$, $3.66\left(1 \mathrm{H}, \mathrm{br} \mathrm{t}, J=5.0 \mathrm{~Hz},-\mathrm{OCH}_{2} \mathrm{CH}_{2} \mathrm{O}-\right), 4.05\left(2 \mathrm{H}, \mathrm{br} \mathrm{t}, J=5.0 \mathrm{~Hz},-\mathrm{OCH}_{2} \mathrm{CH}_{2} \mathrm{O}-\right), 4.60(1 \mathrm{H}, \mathrm{dq}, J=$ 17.0, $1.7 \mathrm{~Hz},-\mathrm{CH}=\mathrm{CHH}), 4.64(1 \mathrm{H}, \mathrm{dq}, J=10.5,1.5 \mathrm{~Hz},-\mathrm{CH}=\mathrm{CH} H), 7.10-7.13(6 \mathrm{H}, \mathrm{m}, \mathrm{Tr}-\mathrm{H}), 7.23-7.33$ (9H, m, Tr-H), $7.34\left(1 \mathrm{H}, \mathrm{s}\right.$, pyrazole-H); ${ }^{13} \mathrm{C}$ NMR $\left(150 \mathrm{MHz}, \mathrm{CDCl}_{3}\right): \delta 31.2,59.2,71.2,71.4,78.5,115.6$, 125.5, 127.3, 127.6, 127.9, 130.1, 132.6, 143.0, 144.4; HREIMS $m / z$ calcd. for $\mathrm{C}_{28} \mathrm{H}_{28} \mathrm{~N}_{2} \mathrm{O}_{2}\left(\mathrm{M}^{+}\right)$424.2151, found 424.2157 .

\subsection{RCM of $13 \mathbf{a}$ and $14 \mathbf{a}$ and $14 \mathbf{b}$}

The RCM reactions of $13 \mathbf{a}$ and $14 \mathbf{a}$ and $\mathbf{1 4 b}$ in Scheme 2 were carried out as described above.

1,7-Dihydro-1-tritylpyrano[3,2-c]pyrazole (8a): oil; IR (film) v $v_{\max } 1583$ (C=C), 1493 (C=C), 1446 $(\mathrm{C}=\mathrm{C}) \mathrm{cm}^{-1} ;{ }^{1} \mathrm{H}$ NMR $\left(600 \mathrm{MHz}, \mathrm{CDCl}_{3}\right): \delta 2.27\left(2 \mathrm{H}, \mathrm{dd}, J=3.5,2.0 \mathrm{~Hz}, \mathrm{ArCH}_{2} \mathrm{CH}=\mathrm{CH}-\right), 4.49(1 \mathrm{H}$, $\left.\mathrm{dt}, J=6.5,3.5 \mathrm{~Hz},-\mathrm{OCH}=\mathrm{CHCH}_{2}-\right), 6.33\left(1 \mathrm{H}, \mathrm{dt}, J=6.4,2.0 \mathrm{~Hz},-\mathrm{OCH}=\mathrm{CHCH}_{2}-\right), 7.12-7.15(6 \mathrm{H}, \mathrm{m}$, $\mathrm{Tr}-\mathrm{H}), 7.26-7.32(9 \mathrm{H}, \mathrm{m}, \mathrm{Tr}-\mathrm{H}), 7.32\left(1 \mathrm{H}, \mathrm{s}\right.$, pyrazole-H); ${ }^{13} \mathrm{C} \mathrm{NMR}\left(150 \mathrm{MHz}, \mathrm{CDCl}_{3}\right): \delta 22.5,29.7,78.6$, 98.2, 124.3, 127.6, 127.6, 127.9, 130.4, 140.3, 142.6; HREIMS $m / z$ calcd. for $\mathrm{C}_{25} \mathrm{H}_{20} \mathrm{~N}_{2} \mathrm{O}\left(\mathrm{M}^{+}\right) 364.1575$, found 364.1576 .

\subsection{Acid-Catalyzed Hydrolysis of $\mathbf{6 a}$ (Scheme 3)}

To a solution of $6 \mathbf{a}(121.5 \mathrm{mg}, 0.30 \mathrm{mmol})$ in acetone $(10 \mathrm{~mL})$ was added $1 \mathrm{~N}$ aqueous $\mathrm{HCl}(0.6 \mathrm{~mL})$. The reaction mixture was warmed under reflux for $90 \mathrm{~min}$ with stirring. After the reaction mixture had cooled, it was treated with saturated aqueous $\mathrm{NaHCO}_{3}$ and extracted with $\mathrm{CH}_{2} \mathrm{Cl}_{2}$. The separated organic layer was dried over $\mathrm{MgSO}_{4}$, filtered, and condensed under reduced pressure to give a crude residue, which was purified using silica gel column chromatography (eluent: EtOAc:hexane $=1: 2$ ) to afford $(Z)-\mathbf{1 8}(9.1 \mathrm{mg}, \mathbf{2 0} \%)$ and $(E)-\mathbf{1 8}(14.2 \mathrm{mg}, 31 \%)$.

(E)-4-Allyloxy-5-(1-propenyl)-1H-pyrazole ((E)-18): oil; IR (film) $v_{\max } 1568$ (C=C), 1516 (C=C) $\mathrm{cm}^{-1} ;{ }^{1} \mathrm{H}$ NMR $\left(600 \mathrm{MHz}, \mathrm{CDCl}_{3}\right): \delta 1.89\left(3 \mathrm{H}, \mathrm{br} \mathrm{d}, J=6.0 \mathrm{~Hz}, \mathrm{CH}_{3} \mathrm{CH}=\right), 4.60(2 \mathrm{H}, \mathrm{dt}, J=5.5,1.5 \mathrm{~Hz}$, $\left.-\mathrm{OCH}_{2} \mathrm{CH}=\mathrm{CH}_{2}\right), 4.46(1 \mathrm{H}, \mathrm{dq}, J=10.0,1.5 \mathrm{~Hz},-\mathrm{CH}=\mathrm{CHH}), 5.40(1 \mathrm{H}, \mathrm{dq}, J=17.3,1.5 \mathrm{~Hz},-\mathrm{CH}=\mathrm{CHH})$, $6.04\left(1 \mathrm{H}, \mathrm{ddt}, J=17.3,10.5,5.5 \mathrm{~Hz},-\mathrm{OCH}_{2} \mathrm{CH}=\mathrm{CH}_{2}\right), 6.34(1 \mathrm{H}, \mathrm{d}, J=16.7 \mathrm{~Hz}, \mathrm{ArCH}=\mathrm{CH}-), 6.35-6.41$ $\left(1 \mathrm{H}, \mathrm{m},-\mathrm{CH}=\mathrm{CHCH}_{3}\right), 7.22\left(1 \mathrm{H}, \mathrm{s}\right.$, pyrazole-H); ${ }^{13} \mathrm{C} \mathrm{NMR}\left(150 \mathrm{MHz}, \mathrm{CDCl}_{3}\right): \delta 18.9,72.7,117.7,118.7$, 127.6, 133.4, 142.0; HREIMS $m / z$ calcd. for $\mathrm{C}_{9} \mathrm{H}_{12} \mathrm{~N}_{2} \mathrm{O}\left(\mathrm{M}^{+}\right)$164.0950, found 164.0950.

(Z)-4-Allyloxy-5-(1-propenyl)-1H-pyrazole ((Z)-18): oil; IR (film) $v_{\max } 1570$ (C=C), 1524 (C=C), $1450(\mathrm{C}=\mathrm{C}) \mathrm{cm}^{-1} ;{ }^{1} \mathrm{H}$ NMR $\left(600 \mathrm{MHz}, \mathrm{CDCl}_{3}\right): \delta 1.98\left(3 \mathrm{H}, \mathrm{dd}, J=7.0,1.8 \mathrm{~Hz}, \mathrm{CH}_{3} \mathrm{CH}=\right), 3.49(3 \mathrm{H}$, $\left.\mathrm{s},-\mathrm{OCH}_{3}\right), 4.45\left(2 \mathrm{H}, \mathrm{dt}, J=5.2,1.5 \mathrm{~Hz},-\mathrm{OCH}_{2} \mathrm{CH}=\mathrm{CH}_{2}\right), 5.27(1 \mathrm{H}, \mathrm{dq}, J=10.6,1.5 \mathrm{~Hz},-\mathrm{CH}=\mathrm{CHH})$, $5.38(1 \mathrm{H}, \mathrm{dq}, J=17.0,1.5 \mathrm{~Hz},-\mathrm{CH}=\mathrm{CHH}), 5.82\left(1 \mathrm{H}, \mathrm{dq}, J=11.4,7.0 \mathrm{~Hz},-\mathrm{CH}=\mathrm{CHCH}_{3}\right), 6.03(1 \mathrm{H}, \mathrm{ddt}$, $\left.J=17.3,10.6,5.3 \mathrm{~Hz},-\mathrm{OCH}_{2} \mathrm{CH}=\mathrm{CH}_{2}\right), 6.27\left(1 \mathrm{H}, \mathrm{dq}, J=11.5,1.5 \mathrm{~Hz}, \mathrm{ArCH}=\mathrm{CHCH}_{3}\right), 7.27(1 \mathrm{H}, \mathrm{s}$, pyrazole-H); ${ }^{13} \mathrm{C}$ NMR $\left(150 \mathrm{MHz}, \mathrm{CDCl}_{3}\right): \delta 69.1,69.3,117.7,118.7,127.6,133.4,142.0 ;$ HREIMS $\mathrm{m} / \mathrm{z}$ calcd. for $\mathrm{C}_{9} \mathrm{H}_{12} \mathrm{~N}_{2} \mathrm{O}\left(\mathrm{M}^{+}\right)$164.0950, found 164.0949. 


\subsection{Reprotection of $\mathbf{1 8}$ (Scheme 3)}

General procedure: To a stereo mixture of $(E / Z)-\mathbf{1 8}(15.9 \mathrm{mg}, 0.10 \mathrm{mmol})$ in $\mathrm{CH}_{2} \mathrm{Cl}_{2}(10 \mathrm{~mL})$ were added $\mathrm{TrCl}(43.0 \mathrm{mg}, 0.15 \mathrm{mmol})$ and $\mathrm{Et}_{3} \mathrm{~N}(0.022 \mathrm{~mL}, 0.15 \mathrm{mmol})$ at $0{ }^{\circ} \mathrm{C}$. The reaction mixture was stirred at rt overnight, and then quenched with saturated aqueous $\mathrm{NH}_{4} \mathrm{Cl}$ and extracted with $\mathrm{CH}_{2} \mathrm{Cl}_{2}$. The organic layer was dried over $\mathrm{MgSO}_{4}$, filtered, and condensed under reduced pressure to give a crude residue, which was purified using silica gel column chromatography (eluent: EtOAc:hexane = 1:4) to afford 19 a $(28.9 \mathrm{mg}, 68 \%)$ as an oil.

(E/Z)-4-Allyloxy-3-(1-propenyl)-1H-1-tritylpyrazole (19a): oil; IR (film) $v_{\max } 1560$ (C=C), 1491 $(\mathrm{C}=\mathrm{C}), 1445(\mathrm{C}=\mathrm{C}) \mathrm{cm}^{-1},{ }^{1} \mathrm{H}$ NMR of $(E)$-isomer $\left(600 \mathrm{MHz}, \mathrm{CDCl}_{3}\right): \delta 1.90(3 \mathrm{H}, \mathrm{dd}, J=7.1,1.8 \mathrm{~Hz}$, $\left.\mathrm{CH}_{3} \mathrm{CH}=\mathrm{CH}-\right), 4.27\left(2 \mathrm{H}, \mathrm{dt}, J=5.6,1.5 \mathrm{~Hz},-\mathrm{OCH}_{2} \mathrm{CH}=\mathrm{CH}_{2}\right), 5.20(1 \mathrm{H}, \mathrm{ddd}, J=10.6,3.2,1.5 \mathrm{~Hz}$, $\left.-\mathrm{CH}_{2} \mathrm{CH}=\mathrm{CHH}\right), 5.28\left(1 \mathrm{H}, \mathrm{ddd}, J=17.0,3.2,1.8 \mathrm{~Hz},-\mathrm{CH}_{2} \mathrm{CH}=\mathrm{CHH}\right), 5.75(1 \mathrm{H}, \mathrm{dq}, J=11.5,7.1 \mathrm{~Hz}$, $\left.-\mathrm{CH}=\mathrm{CHCH}_{3}\right), 5.95\left(1 \mathrm{H}, \mathrm{ddt}, J=17.3,10.7,5.6 \mathrm{~Hz},-\mathrm{OCH}_{2} \mathrm{CH}=\mathrm{CH}_{2}\right), 6.29(1 \mathrm{H}, \mathrm{dq}, J=11.5,1.5 \mathrm{~Hz}$, $\left.\mathrm{ArCH}=\mathrm{CHCH}_{3}\right), 6.84\left(1 \mathrm{H}, \mathrm{s}\right.$, pyrazole-H), 7.14-7.18 (6H, m, Tr-H), 7.26-7.30 (9H, m, Tr-H); ${ }^{13} \mathrm{C}$ NMR $\left(150 \mathrm{MHz}, \mathrm{CDCl}_{3}\right): \delta(14.2), 15.6,(60.4), 72.9,78.6,(117.4), 117.6,117.7,127.4,127.5,(127.6), 127.9,130.4$, 133.3, (138.6), (142.0), 143.4, signals in parentheses correspond to some of those of the (Z)-isomer; HREIMS $m / z$ calcd. for $\mathrm{C}_{28} \mathrm{H}_{26} \mathrm{~N}_{2} \mathrm{O}\left(\mathrm{M}^{+}\right)$406.2045, found 406.2040.

(E)-4-Allyloxy-1-benzyl-3-(1-propenyl)-1H-pyrazole (19b): oil; IR (film) $v_{\max } 1566$ (C=C), 1496 $(\mathrm{C}=\mathrm{C}), 1445(\mathrm{C}=\mathrm{C}) \mathrm{cm}^{-1},{ }^{1} \mathrm{H}$ NMR $\left(400 \mathrm{MHz}, \mathrm{CDCl}_{3}\right): \delta 1.87\left(3 \mathrm{H}, \mathrm{dd}, J=6.3,1.2 \mathrm{~Hz}, \mathrm{CH}_{3} \mathrm{CH}=\mathrm{CH}-\right)$, $4.35\left(2 \mathrm{H}, \mathrm{dt}, J=5.4,1.5 \mathrm{~Hz},-\mathrm{OCH}_{2} \mathrm{CH}=\mathrm{CH}_{2}\right), 5.16\left(2 \mathrm{H}, \mathrm{s}, \mathrm{ArCH}_{2} \mathrm{Ph}\right), 5.24(1 \mathrm{H}, \mathrm{dq}, J=10.5$, $\left.1.5 \mathrm{~Hz},-\mathrm{CH}_{2} \mathrm{CH}=\mathrm{CHH}\right), 5.36\left(1 \mathrm{H}, \mathrm{dq}, J=17.2,1.6 \mathrm{~Hz},-\mathrm{CH}_{2} \mathrm{CH}=\mathrm{CHH}\right), 6.00(1 \mathrm{H}, \mathrm{ddt}, J=17.2,10.5$, $\left.5.4 \mathrm{~Hz},-\mathrm{OCH}_{2} \mathrm{CH}=\mathrm{CH}_{2}\right), 6.40\left(1 \mathrm{H}, \mathrm{br} \mathrm{d}, J=16.3 \mathrm{~Hz}, \mathrm{ArCH}=\mathrm{CHCH}_{3}\right), 6.53(1 \mathrm{H}, \mathrm{dq}, J=16.3,6.3 \mathrm{~Hz}$, $\left.\mathrm{ArCH}=\mathrm{CHCH}_{3}\right), 6.92(1 \mathrm{H}, \mathrm{s}$, pyrazole-H), $7.18(2 \mathrm{H}, \mathrm{br} \mathrm{d}, J=8.0 \mathrm{~Hz}, \mathrm{Ph}-\mathrm{H}), 7.26-7.35(3 \mathrm{H}, \mathrm{m}, \mathrm{Ph}-\mathrm{H})$; ${ }^{13} \mathrm{C}$ NMR $\left(100 \mathrm{MHz}, \mathrm{CDCl}_{3}\right): \delta 18.9,56.5,72.6,114.7,117.6,121.4,127.4,127.9,128.7,133.2,136.8,138.4$, 143.1; HREIMS $m / z$ calcd. for $\mathrm{C}_{16} \mathrm{H}_{18} \mathrm{~N}_{2} \mathrm{O}\left(\mathrm{M}^{+}\right)$254.1419, found 254.1416.

\subsection{1. $R C M$ of $\mathbf{1 9}$}

The RCM reactions of $\mathbf{1 9}$ were carried out in a similar manner to that described above to afford 20. 2,5-Dihydro-2-tritylpyrano[3,2-c]pyrazole (20a): oil; IR (film) $v_{\max } 1492$ (C=C), 1447 (C=C) cm ${ }^{-1}$; ${ }^{1} \mathrm{H}$ NMR $\left(400 \mathrm{MHz}, \mathrm{CDCl}_{3}\right): \delta 4.76\left(2 \mathrm{H}, \mathrm{dd}, J=2.9,1.9 \mathrm{~Hz},-\mathrm{OCH}_{2} \mathrm{CH}=\mathrm{CH}-\right), 5.72(1 \mathrm{H}, \mathrm{dt}, J=10.2$, $\left.3.5 \mathrm{~Hz},-\mathrm{OCH}_{2} \mathrm{CH}=\mathrm{CH}-\right), 6.62\left(1 \mathrm{H}, \mathrm{br} \mathrm{d}, J=10.0 \mathrm{~Hz},-\mathrm{OCH}_{2} \mathrm{CH}=\mathrm{CHAr}\right), 6.80(1 \mathrm{H}, \mathrm{s}$, pyrazole-H), 7.18-7.20 (6H, m, Tr-H), 7.28-7.31 (9H, m, Tr-H); ${ }^{13} \mathrm{C}$ NMR $\left(150 \mathrm{MHz}, \mathrm{CDCl}_{3}\right): \delta 67.2,78.0,116.5$, 120.1, 122.7, 126.5, 127.6, 127.7, 137.5, 139.1, 143.3; HREIMS $m / z$ calcd. for $\mathrm{C}_{25} \mathrm{H}_{20} \mathrm{~N}_{2} \mathrm{O}\left(\mathrm{M}^{+}\right)$364.1575, found 364.1584 .

2-Benzyl-2,5-dihydropyrano[3,2-c]pyrazole (20b): oil; IR (film) $v_{\max } 1660(\mathrm{C}=\mathrm{C}), 1576(\mathrm{C}=\mathrm{C}) \mathrm{cm}^{-1}$; ${ }^{1} \mathrm{H}$ NMR $\left(400 \mathrm{MHz}, \mathrm{CDCl}_{3}\right): \delta 4.77\left(2 \mathrm{H}, \mathrm{dd}, J=3.5,1.9 \mathrm{~Hz},-\mathrm{OCH}_{2} \mathrm{CH}=\mathrm{CH}-\right), 5.73(1 \mathrm{H}, \mathrm{dt}, J=10.0$, $\left.3.5 \mathrm{~Hz},-\mathrm{OCH}_{2} \mathrm{CH}=\mathrm{CH}-\right), 6.63\left(1 \mathrm{H}, \mathrm{dt}, J=10.0,1.9 \mathrm{~Hz},-\mathrm{OCH}_{2} \mathrm{CH}=\mathrm{CHAr}\right), 6.84(1 \mathrm{H}, \mathrm{s}$, pyrazole- $\mathrm{H})$, 7.18-7.20 (2H, br d, $J=6.5 \mathrm{~Hz}, \mathrm{Ph}-\mathrm{H}), 7.27-7.36(3 \mathrm{H}, \mathrm{m}, \mathrm{Ph}-\mathrm{H}) ;{ }^{13} \mathrm{C}$ NMR $\left(100 \mathrm{MHz}, \mathrm{CDCl}_{3}\right): \delta 56.4$, $67.2,113.3,119.5,122.3,127.5,128.0,128.8,136.6,137.2,140.5 ;$ HREIMS $m / z$ calcd. for $\mathrm{C}_{13} \mathrm{H}_{12} \mathrm{~N}_{2} \mathrm{O}\left(\mathrm{M}^{+}\right)$ 212.0949, found 212.0950.

Author Contributions: Y.U. and K.S. conceived and designed the experiments; K.S., A.K., Y.T., and Y.U. performed the experiments; Y.U., H.Y., and S.H. wrote the paper.

Funding: This research received no external funding.

Acknowledgments: The authors are grateful to K. Minoura and M. Fujitake for NMR and MS measurements, respectively. Y. Suzuki, R. Nakamura, K. Hashimoto, and H. Matsukawa of our laboratory group are also appreciated for their experimental assistance. We would like to thank Editage (www.editage.jp) for English language editing.

Conflicts of Interest: The authors declare no conflicts of interest. 


\section{References}

1. Ansari, A.; Ali, A.; Asif, M.; Shamsuzzaman. Review: Biologically Active Pyrazole Derivatives. New J. Chem. 2017, 41, 16-41. [CrossRef]

2. Küçükgüzel, Ş.G.; Şenkardeş, S. Recent Advances in Bioactive Pyrazoles. Eur. J. Med. Chem. 2015, 97, 786-815. [CrossRef]

3. Li, J.J. Pyrazoles, Pyrazolones, and Indazoles. In Heterocyclic Chemistry in Drug Discovery; Wiley-VCH: Weinheim, Germany, 2013; pp. 198-229. ISBN 978-1-118-14890-7.

4. Fustero, S.; Sanchez-Rosello, M.; Barrio, P.; Simon-Fuentes, A. From 2000 to Mid-2010: A Fruitful Decade for the Synthesis of Pyrazoles. Chem. Rev. 2011, 111, 6984-7034. [CrossRef]

5. Ichikawa, H.; Ohno, Y.; Usami, Y.; Arimoto, M. Synthesis of 4-Arylpyrazoles via $\mathrm{PdCl}_{2}$ (dppf)-Catalyzed Cross Coupling Reaction with Grignard Reagents. Heterocycles 2006, 68, 2247-2252. [CrossRef]

6. Ichikawa, H.; Nishioka, M.; Arimoto, M.; Usami, Y. Synthesis of 4-Aryl-1H-pyrazoles by Suzuki-Miyaura Cross Coupling Reaction between 4-Bromo-1H-1-tritylpyrazole and Arylboronic Acids. Heterocycles 2010, 81, 1509-1516. [CrossRef]

7. Usami, Y.; Ichikawa, H.; Harusawa, S. Heck-Mizoroki Reaction of 4-Iodo-1H-pyrazoles. Heterocycles 2011, 83, 827-835. [CrossRef]

8. Ichikawa, H.; Ohfune, H.; Usami, Y. Microwave-Assisted Selective Synthesis of 2H-Indazoles via Double Sonogashira Coupling of 3,4-Diiodopyrazoles and Bergman-Masamune Cycloaromatization. Heterocycles 2010, 81, 1651-1659. [CrossRef]

9. Li, M.; Zhao, B.-X. Progress of the Synthesis of Condensed Pyrazole Derivatives (From 2010 to Mid-2013). Eur. J. Med. Chem. 2014, 85, 311-340. [CrossRef]

10. Das, D.; Banerjee, R.; Mitra, A. Bioactive and Pharmacologically Important Pyrano[2,3-c]pyrazoles. J. Chem. Pharm. Res. 2014, 6, 108-116.

11. Ueda, T.; Mase, H.; Oda, N.; Ito, I. Synthesis of Pyrazolone Derivatives. XXXIX. Synthesis and Analgesic Activity of Pyrano[2,3-c]pyrazoles. Chem. Pharm. Bull. 1981, 29, 3522-3528. [CrossRef]

12. Huang, L.-J.; Hour, M.-J.; Teng, C.-M.; Kuo, S.-C. Synthesis and Antiplatelet Activities of N-Arylmethyl-3,4-dimethylpyrano[2,3-c]pyrazol-6-one Derivatives. Chem. Pharm. Bull. 1992, 40, 2547-2551. [CrossRef]

13. Adibi, H.; Hosseinzadeh, L.; Farhadi, S.; Ahmadi, F. Synthesis and Cytotoxic Evaluation of 6-Amino-4-aryl-3-methyl-2,4-dihydropyrano[2,3-c]pyrazole-Carbonitrile Derivatives Using Borax with Potential Anticancer Effects. J. Rep. Pharm. Sci. 2013, 2, 116-125.

14. Kashtoh, H.; Muhammad, M.T.; Khan, J.J.; Rasheed, S.; Khan, A.; Perveen, S.; Javaid, K.; Atia-tul-Wahab; Khan, K.M.; Choudhary, M.I. Dihydropyrano [2,3-c] Pyrazole: Novel in vitro Inhibitors of Yeast $\alpha$-Glucosidase. Bioorg. Chem. 2016, 65, 61-72. [CrossRef]

15. Freeman-Cook, K.D.; Amor, P.; Bader, S.; Buzon, L.M.; Coffey, S.B.; Corbett, J.W.; Dirico, K.J.; Doran, S.D.; Elliott, R.L.; Esler, W.; et al. Maximizing Lipophilic Efficiency: The Use of Free-Wilson Analysis in the Design of Inhibitors of Acetyl-CoA Carboxylase. J. Med. Chem. 2012, 55, 935-942. [CrossRef]

16. Chou, L.-C.; Huang, L.-J.; Yang, J.-S.; Lee, F.-Y.; Teng, C.-M.; Kuo, S.-C. Synthesis of Furopyrazole Analogs of 1-Benzyl-3-(5-hydroxymethyl-2-furyl)indazole (YC-1) as Novel Anti-Leukemia Agents. Bioorg. Med. Chem. 2007, 15, 1732-1740. [CrossRef]

17. Maggio, D.; Raffa, M.V.; Raimondi, F.; Plescia, M.L.; Trincavelli, C.; Martini, F.; Meneghetti, L.; Basile, S.; Guccione, G.D. Synthesis, Benzodiazepine Receptor Binding and Molecular Modelling of Isochromeno[4,3-c]pyrazol-5(1H)-one Derivatives. Eur. J. Med. Chem. 2012, 54, 709-720. [CrossRef]

18. Lin, Y.-C.; Chou, L.-C.; Chen, S.-C.; Kuo, S.-C.; Huang, L.-J.; Gean, P.-W. Neuroprotective Effects of Furopyrazole Derivative of Benzylindazole Analogs on C2 Ceramide-Induced Apoptosis in Cultured Cortical Neurons. Bioorg. Med. Chem. Lett. 2009, 19, 3225-3228. [CrossRef]

19. Blanchard, N.; Eustache, J. Synthesis of Natural Products Containing Medium-Size Carbocycles by Ring-Closing Alkene Metathesis. In Metathesis in Natural Product Synthesis; Cossy, J., Arseniyadis, S., Meyer, C., Eds.; Wiley-VCH Verlag GmbH \& Co. KGaA: Weinheim, Germany, 2010; pp. 1-43. ISBN 978-3-527-32440-8.

20. Kotha, S.; Krishna, N.G.; Halder, S.; Misra, S. Synergistic approach to polycyclics via a strategic utilization of Claisen rearrangement and olefin metathesis. Org. Biomol. Chem. 2011, 9, 5597-5624. [CrossRef] 
21. Ichikawa, H.; Watanabe, R.; Fujino, Y.; Usami, Y. Divergent Synthesis of Withasomnines via Synthesis of 4-Hydroxy-1H-pyrazoles and Claisen Rearrangement of Their 4-O-Allylethers. Tetrahedron Lett. 2011, 52, 4448-4451. [CrossRef]

22. Usami, Y.; Watanabe, R.; Fujino, Y.; Shibano, M.; Ishida, C.; Yoneyama, H.; Harusawa, S.; Ichikawa, H. Divergent Synthesis and Evaluation of Inhibitory Activities Against Cyclooxygenases-1 and -2 of Natural Withasomnines and Analogues. Chem. Pharm. Bull. 2012, 60, 1550-1560. [CrossRef]

23. Usami, Y.; Kohno, A.; Yoneyama, H.; Harusawa, S. Synthesis of Dihydrooxepino[3,2-c]pyrazoles via Claisen Rearrangement and Ring Closing Metathesis from 4-Allyloxy-1H-pyrazoles. Molecules 2018, $23,592$. [CrossRef]

24. Zolfigol, M.A.; Navazeni, M.; Yarie, M.; Ayazi-Nasrabadi, R. Application of a Biological-Based Nanomagnetic Catalyst in the Synthesis of Bis-pyrazoles and Pyrano[3,2-c] Pyrazoles. Appl. Organomet. Chem. 2017, 31, e3633. [CrossRef]

25. Hanamono, T.; Hashimoto, E.; Miura, M.; Furuno, H.; Inanaga, J. Reaction of N-Methyl-5-tributylstannyl4-fluoro- $1 \mathrm{H}$-pyrazole and Its Application to N-Methyl-chromeno[2,3- $d$ ]pyrazol-9-one Synthesis. J. Org. Chem. 2008, 73, 4736-4739. [CrossRef]

26. Huang, K.S.; Li, S.R.; Wang, Y.F.; Lin, Y.L.; Chen, Y.H.; Tsai, T.W.; Yang, C.H.; Wang, E.C. Synthesis of Certain Benzoheterocyclic Compounds from 2-Hydroxyacetophenone via Cyclization and Ring-Closing Metathesis. J. Chin. Chem. Soc. 2005, 52, 159-167. [CrossRef]

27. Wakamatsu, H.; Nishida, M.; Adachi, N.; Mori, M. Isomerization Reaction of Olefin Using $\mathrm{RuClH}(\mathrm{CO})\left(\mathrm{PPh}_{3}\right)_{3}$. J. Org. Chem. 2000, 63, 3966-3970. [CrossRef]

Sample Availability: Samples of the compounds are not available from the authors.

(C) 2019 by the authors. Licensee MDPI, Basel, Switzerland. This article is an open access article distributed under the terms and conditions of the Creative Commons Attribution (CC BY) license (http://creativecommons.org/licenses/by/4.0/). 